\title{
Workspace Tracking Control of Two-Flexible-Link Manipulator Using Distributed Control Strategy
}

\author{
Fareh Raouf, ${ }^{1}$ Saad Mohamad, ${ }^{2}$ and Saad Maarouf ${ }^{1}$ \\ ${ }^{1}$ Electrical Engineering Department, University of Quebec, École de Technologie Supérieure, 1100 Notre-Dame West, Montreal, \\ QC, Canada H3C $1 K 3$ \\ ${ }^{2}$ School of Engineering, University of Quebec at Abitibi-Témiscamingue, 445 University, Rouyn-Noranda, \\ QC, Canada J9X 5E4
}

Correspondence should be addressed to Fareh Raouf; raouf.fareh.1@ens.etsmtl.ca

Received 5 April 2013; Accepted 25 September 2013

Academic Editor: Mohamed Zribi

Copyright ( $\odot 2013$ Fareh Raouf et al. This is an open access article distributed under the Creative Commons Attribution License, which permits unrestricted use, distribution, and reproduction in any medium, provided the original work is properly cited.

A distributed nonlinear control strategy for two-flexible-link manipulators is presented to track a desired trajectory in the robot's workspace. The inverse dynamics problem is solved by transforming the desired trajectory from the workspace to the joint space using an intermediate space, called virtual space, and then using the quasi-static approach. To solve the nonminimum phase problem, an output redefinition technique is used. This output consists of the motor's angle augmented with a weighted value of the link's extremity. The distributed control strategy consists in controlling the last link by assuming that the first link is stable and follows its desired trajectories. The control law is developed to stabilize the error dynamics and to guarantee bounded internal dynamics such that the new output is as close as possible to the tip. The weighted parameter defining the noncollocated output is then selected. The same procedure is applied to control and stabilize the first link. The asymptotical stability is proved using Lyapunov theory. This algorithm is applied to a two-flexible-link manipulator in the horizontal plane, and simulations showed a good tracking of the desired trajectory in the workspace.

\section{Introduction}

Many control strategies for manipulators have been the focus of several studies in recent years. The robot manipulators consist of a sequence of links and joints in various combinations. In industrial applications, most of the existing manipulators use rigid links and joints and are known as rigid manipulators. Rigid manipulators are generally slow, extremely rigid, and massive, and the useful load is very low compared to their weight. To improve the performance of the robot manipulators, their links must be lighter, and therefore they become more flexible. Flexible manipulators present more advantages when compared to rigid manipulators: they are faster and less massive and consume less energy. Some flexible manipulators are used in different areas, for example, the aerospace applications [1] and medical applications [2].

For flexible manipulators, the problem of workspace tracking trajectory is less covered so far than that of joint space tracking. There are few solutions to the workspace tracking problem, particularly for manipulators with many flexible links. The workspace tracking trajectory is very important since most of the tasks are defined in the operational space, such as painting, welding, and assembly. The flexible link manipulators are a nonminimum phase system when controlling the position of the end effector [3]. Unlike rigid manipulators, the inverse kinematics of flexible manipulators is not sufficient to transform the desired trajectory from workspace to joint space because they are linked by kinematics and dynamics relationships. To solve this problem, many studies focused on the output redefinition technique [4-7]. This technique consists in selecting a new output as close as possible to the tip such that internal dynamics become bounded. In [5], this approach was used for a class of manipulators where the last link is flexible. An intermediate space between the joint space and the workspace called virtual space is used to transform the desired trajectory from the workspace to the joint space. The virtual space is linked with the workspace by a simple kinematics relation as in rigid 
manipulators. In [4], the output redefinition technique was used for one flexible link manipulator. This output consists of the motor's angle augmented with a weighted value of the angle of links extremity.

The nonlinear dynamics of flexible link manipulators combined with their under-actuated nature (the deflection variables are not actuated) present a challenging control problem. Multi-flexible-link manipulators can be controlled as one MIMO system so a single controller is used for all joints and links or as a set of interconnected subsystems so each pair of joint and link is controlled by its own controller. For the first case, many control schemes were used. Several studies used linearization around a nominal configuration of flexible manipulators model $[8,9]$. Some nonlinear effects such as the variation of inertia matrix around an operating point were taken into account in the control design methodology. In [10], a control method based on the input-output linearization was developed to track a desired trajectory in the workspace for a class of flexible link manipulators when the last link is flexible. To select an output near the tip that guarantees the stability of the zero dynamics, authors used the output redefinition technique. A robust adaptive controller was developed in [11] for a class of flexible link manipulators where the last link is flexible. This controller is based on feedback linearization and uses the virtual joint space that is kinematically related to workspace. For trajectory tracking control problems, many other techniques were covered in the literature such as singular perturbation technique [12], sliding mode control [13], inversion-based nonlinear control [14], and so forth. All the above-mentioned control methods use a single controller for all joints and links as one MIMO system. Unfortunately, due to the complexity of the control structures, the real-time implementation in industrial applications is not easy [15]. A solution to this problem can be followed by considering the dynamics of the robot manipulators as interconnected subsystems (joint and link). Many control schemes consider this configuration. In [16], a decentralized control method for flexible link manipulators was used. The authors used a simple proportional derivative (PD) controller for the joints and a linear quadratic regulator (LQR) with output feedback for each link. In [17], a PD controller was used for each joint and the measurement of linear velocity of the tip position was used for controlling each link. A distributed control strategy was introduced in $[18,19]$ for rigid link manipulators. The distributed control strategy consists in controlling one joint at a time starting with the last joint and going backward until the first one. Lyapunov theory was used to prove the global stability of the error dynamics, and this controller was successfully applied on a 7 DOF manipulator. This control strategy was modified in [20] to take into account links' flexibility. A good tracking performance in the workspace of a two-flexiblelink manipulator was obtained. However, the control law, based on the feedback linearization approach, ensures only local stability. In this paper, a nonlinear distributed control strategy is presented for a two-flexible-link manipulator that ensures global stability. This strategy consists of controlling the second joint and link by assuming that the remaining joint and link are stable and follow their desired trajectories. Then, going backward, the first joint and link are controlled

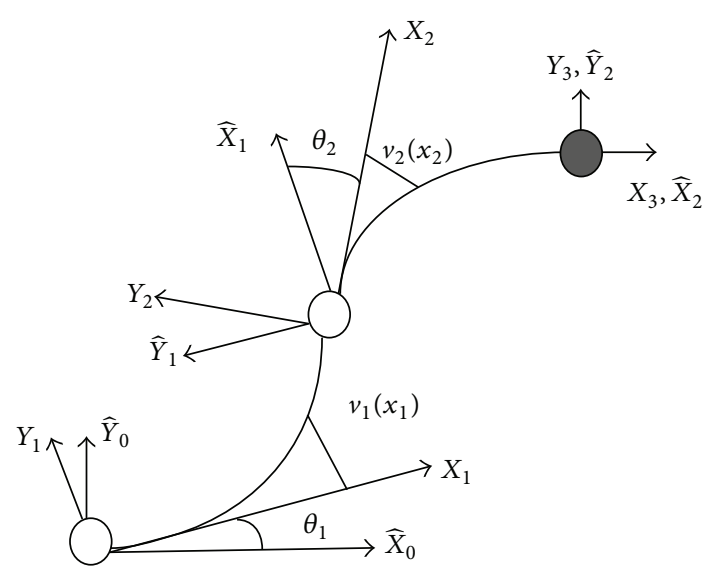

FIGURE 1: Two-flexible-link manipulator.

by following the same strategy. For the inversion dynamics problem, the virtual space and the quasi-static approach are used. The output redefinition technique is used to obtain the nearest point to the tip that ensures bounded internal dynamics. The Lyapunov approach is used to analyze stability of the tracking errors.

The paper is organized as follows. Section 2 presents the modeling of the two-flexible-link manipulator and presents its main properties that will be used in the control law design. Section 3 presents the distributed control strategy. The stability analysis is given in Section 4. The control method is applied on a two-flexible-link manipulator, and the simulations are given in Section 5. Finally, a conclusion is given in Section 6.

\section{Modeling}

2.1. System Description. Figure 1 shows a two-flexible-link manipulator. This system moves in the horizontal plane and consists of two motors that generate two torques, two flexible links with mass $m_{i}$, length $L_{i}$, linear density $\rho_{i}$, and rigidity $E I_{i}(i=1,2)$, and a payload that has a mass $m_{p}$. The first link is attached to the first motor, and the second link is clamped to the rotor of the second motor. The flexible links are supposed uniform and are modeled as Euler-Bernoulli beams, and the deformations are assumed to be small.

Using Lagrange equations, the dynamical model of an $n$ DOF flexible manipulator is given by [21]

$$
M(q) \ddot{q}+H(q, \dot{q})+D \dot{q}+K q=\tau,
$$

where $M$ is the inertia and mass matrix, $H(q, \dot{q})$ is the Coriolis and centrifugal forces vector, $D$ is the friction matrix, and $K$ is the rigidity matrix. $q$ represents the vector of the generalized coordinates and $\tau$ is the vector of the applied torques. For the 
$n$ rigid coordinates and $n$ flexible links, the deformation of the $i$ th flexible link is given by the following equation:

$$
v_{i}(x, t)=\sum_{j=1}^{z_{i}} \emptyset_{i j}(x) q_{f i j}(t), \quad i=1, \ldots, n,
$$

where $q_{f i j}$ is the $j$ th generalized flexible coordinate, $\emptyset_{i j}(x)$ is its $j$ th shape function, and $z_{i}$ is the number of the retained flexible modes of the $i$ th flexible link. Note that the total number of the flexible modes is $z=\sum_{i=1}^{n} z_{i}$ and the number of the rigid modes is $n$. The dynamical model (1) can be written as follows:

$$
\begin{aligned}
& {\left[\begin{array}{cc}
M_{r r} & M_{r f} \\
M_{r f}^{T} & M_{f f}
\end{array}\right]\left[\begin{array}{l}
\ddot{q}_{r} \\
\ddot{q}_{f}
\end{array}\right]+\left[\begin{array}{l}
H_{r}(q, \dot{q}) \\
H_{f}(q, \dot{q})
\end{array}\right]} \\
& \quad+\left[\begin{array}{cc}
0 & 0 \\
0 & D_{f f}
\end{array}\right]\left[\begin{array}{c}
\dot{q}_{r} \\
\dot{q}_{f}
\end{array}\right]+\left[\begin{array}{cc}
0 & 0 \\
0 & K_{f f}
\end{array}\right]\left[\begin{array}{l}
q_{r} \\
q_{f}
\end{array}\right]=\left[\begin{array}{l}
I \\
0
\end{array}\right] \tau,
\end{aligned}
$$

where $M_{r r} \in \mathfrak{R}^{n \times n}$ and $M_{f f} \in \mathfrak{R}^{z \times z}$ are mass and inertia matrices for the rigid and flexible part, respectively. $M_{r f} \in$ $\mathfrak{R}^{n \times z}$ is a coupled element. $K_{f f}$ is the stifness diagonal matrix, and $D_{f f}$ is the damping diagonal matrix of the flexible part. The subscripts $r$ and $f$ denote the rigid and flexible modes.

2.2. Proprieties and Problem Formulation. The dynamical model of the flexible link manipulators has the following properties that will be used in the control law development:

(P1) $M, M_{r r}, M_{f f}, D_{f f}$, and $K_{f f}$ are symmetric positive definite matrices [21].

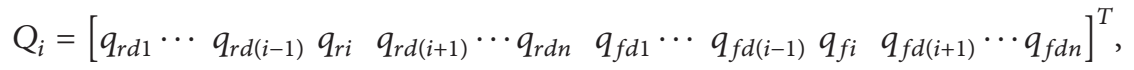

where $\left(q_{r i}, q_{f i}\right)$ are the rigid and flexible modes associated with the $i$ th motor and link, respectively. The objective of this work is to track a desired trajectory defined in the workspace of a two-flexible-link manipulator using distributed control. Three steps are followed to achieve this objective.

Step 1. Transform the desired trajectory from the workspace to the joint space using inverse kinematics and quasi-static approach.

Step 2. Develop the control law for the second and first links to stabilize the error dynamics and to guarantee bounded internal dynamics such that the output is as close as possible to the tip.

Step 3. Study the global stability.
(P2) From (P1), we can deduce that the diagonal elements of $M$ are positive [22]:

$$
M_{i i}(q)>0 ; \quad \text { for } i=1, \ldots, n \text {. }
$$

(P3) There exists a matrix $C(q, \dot{q})$ such that

$$
H(q, \dot{q})=C(q, \dot{q}) \dot{q}
$$

(P4) The inertia-mass matrix $M(q)$ and the Coriolis matrix $C(q, \dot{q})$ satisfy the following skew-symmetric property:

$$
X^{T}(\dot{M}(q, \dot{q})-2 C(q, \dot{q})) X=0, \quad \forall X \in \mathscr{R}^{n+z} .
$$

(P5) The propriety (P4) is preserved for the diagonal elements of $M(q)$ and $C(q, \dot{q})$. Then, we can write

$$
\dot{M}_{i i}(q)-2 C_{i i}(q, \dot{q})=0, \quad \text { for } i=1, \ldots, n \text {. }
$$

The new noncollocated output of the $i$ th link defined by the motor's angle augmented with a weighted value $\alpha_{i}$ of the endpoint angular position is given as follows:

$$
y_{i}=q_{r i}+\alpha_{i} \beta_{i}^{T} q_{f i}, \quad 0 \leq \alpha_{i}<1,
$$

where $\beta_{i}^{T}=\left(1 / L_{i}\right)\left[\emptyset_{i 1}\left(L_{i}\right), \ldots, \emptyset_{i z_{i}}\left(L_{i}\right)\right]$, with $L_{i}$ being the length of the $i$ th flexible link, and $\emptyset_{i j}$ is the shape function of link $i$ and mode $j$. In this paper, we consider only the first flexible mode of each link (i.e., $z_{i}=1$ ). Thus, we have $z=n$.

In the distributed control strategy, when controlling the $i$ th link, we assume that the other links follow their desired trajectories. For the $i$ th link, a new generalized coordinate $Q_{i}$ is defined such that the $i$ th coordinate is the controlled one and the other links follow their desired trajectories such that

\section{Distributed Control Strategy}

3.1. Inverse Dynamics. To achieve the objective of workspace tracking trajectories, we need to transform the desired trajectories from the workspace to the joint space. The flexible manipulator is a nonminimum phase system when the end effector is used as the output. In this case, kinematic and dynamic relationships link the workspace and the joint space. To overcome this problem, an intermediate space called virtual space can be used. Then the desired workspace trajectory is transformed to the virtual space using an inverse kinematics relation as in rigid manipulators. To transform the desired trajectories from the virtual space to the joint pace, the quasi-static approach can be used to solve a nonlinear equation for the flexible part.

Using inverse kinematics as in rigid manipulators, the generalized coordinates in virtual space can be easily found. 


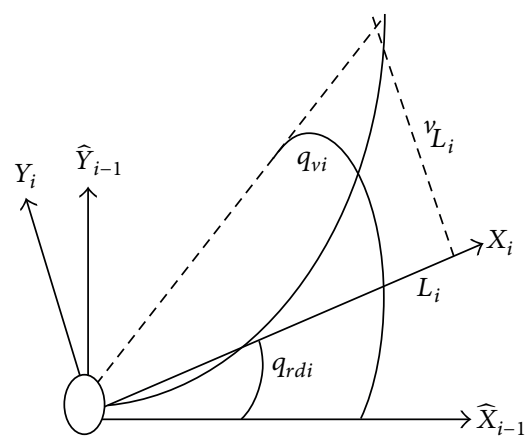

Figure 2: Virtual space.

The deformation is assumed to be small. According to Figure 2, we can write

$$
q_{v i}-q_{r d i}=\arctan \left(\frac{v_{L_{i}}}{L_{i}}\right) \approx \frac{v_{L_{i}}}{L_{i}} .
$$

In the virtual space, the generalized coordinate is given as follows:

$$
q_{v i}=q_{r d i}+\beta_{i} q_{f d i}
$$

where $\beta_{i}=\left(\emptyset_{i}\left(L_{i}\right) / L_{i}\right)$ and $\emptyset_{i}$ is the shape function of link $i$.

Using the Jacobian matrix as in rigid manipulators, the velocity and acceleration in the virtual space can be deduced. Then, for a two-DOF manipulator, the inverse kinematics is given by the following equation:

$$
\begin{gathered}
q_{v}=\left[\begin{array}{l}
q_{v 1} \\
q_{v 2}
\end{array}\right]=\left[\begin{array}{c}
\arctan 2(y, x)-\arctan 2\left(L_{2} s_{2}, L_{1}+L_{2} c_{2}\right) \\
\arctan 2\left(s_{2}, c_{2}\right)
\end{array}\right], \\
\dot{q}_{v}=\left[\begin{array}{c}
\dot{q}_{v 1} \\
\dot{q}_{v 2}
\end{array}\right]=J^{-1}\left(q_{v}\right)\left[\begin{array}{c}
\dot{x} \\
\dot{y}
\end{array}\right], \\
\ddot{q}_{v}=\left[\begin{array}{l}
\ddot{q}_{v 1} \\
\ddot{q}_{v 2}
\end{array}\right]=J^{-1}\left(q_{v}\right)\left[\begin{array}{c}
\ddot{x} \\
\ddot{y}
\end{array}\right]-J^{-1}\left(q_{v}\right) \dot{J}\left(q_{v}\right) \dot{q}_{v},
\end{gathered}
$$

where $x$ and $y$ are the workspace desired positions; $c_{2}=$ $\left(x^{2}+y^{2}-L_{1}^{2}-L_{2}^{2}\right) / 2 L_{1} L_{2} ; s_{2}= \pm \sqrt{1-c_{2}^{2}}$ and $J\left(q_{v}\right)=$ $\left[\begin{array}{cc}-L_{1} s_{1}-L_{2} s_{12} & -L_{2} s_{12} \\ L_{1} c_{1}+L_{2} c_{12} & L_{2} c_{12}\end{array}\right]$ is the Jacobian matrix. $s_{1}=\sin \left(q_{v 1}\right) ; c_{1}=$ $\cos \left(q_{v 1}\right) ; s_{12}=\sin \left(q_{v 1}+q_{v 2}\right) ; c_{12}=\cos \left(q_{v 1}+q_{v 2}\right) ; L_{1}$ is length of link 1 and $L_{2}$ is length of link 2 . The rigid and flexible coordinates are derived via quasi-static approach and using the generalized coordinates in the virtual space.

The dynamical model (3) can be written as a function of the desired coordinates as follows:

$$
\begin{gathered}
M_{r r}\left(q_{d}\right) \ddot{q}_{r d}+M_{r f}\left(q_{d}\right) \ddot{q}_{f d}+H_{r}\left(q_{d}, \dot{q}_{d}\right)=\tau, \\
M_{r f}^{T}\left(q_{d}\right) \ddot{q}_{r d}+M_{f f}\left(q_{d}\right) \ddot{q}_{f d}+H_{f}\left(q_{d}, \dot{q}_{d}\right) \\
+D_{f f} \dot{q}_{f d}+K_{f f} q_{f d}=0 .
\end{gathered}
$$

The generalized flexible and rigid coordinates in the joint space are deduced using the quasi-static approach. In the first step, the nonlinear equation of the flexible part (14) is solved to find the generalized flexible coordinates. The quasi-static approach neglects the desired velocity and acceleration of the flexible coordinates $\left(\dot{q}_{f d}=\ddot{q}_{f d}=0\right)$. Then, the equation of the flexible part (14) can be written as follows:

$$
M_{r f}^{T}\left(q_{d}\right) \ddot{q}_{r d}+H_{f}\left(q_{d}, \dot{q}_{d}\right)+K_{f f} q_{f d}=0,
$$

where

$$
q_{r d i}=q_{v i}-\beta_{i}^{T} q_{f d i} ; \quad \dot{q}_{r d i}=\dot{q}_{v i} ; \quad \ddot{q}_{r d i}=\ddot{q}_{v i} .
$$

Using (16), the generalized flexible coordinates $q_{f d}$ are found by solving (15). Then, the generalized rigid coordinates are given by (16).

3.2. Control Strategy. For two-flexible-link manipulator, the distributed control strategy consists of controlling and stabilizing the last joint and flexible link by assuming that the first joint and flexible link are stable and follow their desired trajectories. Then, we move backward and apply the same procedure to the first joint and link. For each step, a new noncollocated output and a control law are developed. The weighting parameter characterizing the noncollocated output is calculated such that the tracking error is asymptotically stable. Thus, the system becomes a minimum phase with the selected new weighted outputs.

In this paper, the two-flexible-link model given in $[21,23]$ is modified by considering only the first flexible mode of each link [20]. Thus, we have $n=z=2$ and $z_{1}=z_{2}=1$.

Using (P3), the dynamical model of the two-flexible-link manipulator can be written as

$$
M(q) \ddot{q}+C(q, \dot{q}) \dot{q}+D \dot{q}+K q=L \tau,
$$

where

$$
\begin{aligned}
& M=\left[\begin{array}{llll}
M_{11} & M_{12} & M_{13} & M_{14} \\
M_{21} & M_{22} & M_{23} & M_{24} \\
M_{31} & M_{32} & M_{33} & M_{34} \\
M_{41} & M_{42} & M_{43} & M_{44}
\end{array}\right] \\
& C=\left[\begin{array}{llll}
C_{11} & C_{12} & C_{13} & C_{14} \\
C_{21} & C_{22} & C_{23} & C_{24} \\
C_{31} & C_{32} & C_{33} & C_{34} \\
C_{41} & C_{42} & C_{43} & C_{44}
\end{array}\right] ; \\
& D=\left[\begin{array}{cccc}
0 & 0 & 0 & 0 \\
0 & 0 & 0 & 0 \\
0 & 0 & D_{f 1} & 0 \\
0 & 0 & 0 & D_{f 2}
\end{array}\right] \\
& K=\left[\begin{array}{cccc}
0 & 0 & 0 & 0 \\
0 & 0 & 0 & 0 \\
0 & 0 & K_{f 1} & 0 \\
0 & 0 & 0 & K_{f 2}
\end{array}\right] \\
& q=\left[\begin{array}{c}
q_{r 1} \\
q_{r 2} \\
q_{f 1} \\
q_{f 2}
\end{array}\right] ; \quad L=\left[\begin{array}{cc}
1 & 0 \\
0 & 1 \\
0 & 0 \\
0 & 0
\end{array}\right] ; \quad \tau=\left[\begin{array}{l}
\tau_{1} \\
\tau_{2}
\end{array}\right] .
\end{aligned}
$$


In rigid and flexible part decomposition, the dynamical model (17) can be written as

$$
\begin{gathered}
{\left[\begin{array}{cc}
M_{r} & M_{r f} \\
M_{r f}^{T} & M_{f}
\end{array}\right]\left[\begin{array}{l}
\ddot{q}_{r} \\
\ddot{q}_{f}
\end{array}\right]+\left[\begin{array}{cc}
C_{r} & C_{r f} \\
C_{r f}^{T} & C_{f}
\end{array}\right]\left[\begin{array}{l}
\dot{q}_{r} \\
\dot{q}_{f}
\end{array}\right]} \\
+\left[\begin{array}{cc}
0 & 0 \\
0 & D_{f}
\end{array}\right]\left[\begin{array}{l}
\dot{q}_{r} \\
\dot{q}_{f}
\end{array}\right]+\left[\begin{array}{cc}
0 & 0 \\
0 & K_{f}
\end{array}\right]\left[\begin{array}{l}
q_{r} \\
q_{f}
\end{array}\right]=\left[\begin{array}{l}
1 \\
0
\end{array}\right] \tau,
\end{gathered}
$$

where

$$
\begin{array}{rlr}
M_{r}=\left[\begin{array}{ll}
M_{11} & M_{12} \\
M_{21} & M_{22}
\end{array}\right] ; & M_{r f}=\left[\begin{array}{ll}
M_{13} & M_{14} \\
M_{23} & M_{24}
\end{array}\right] ; \\
M_{f}=\left[\begin{array}{ll}
M_{33} & M_{34} \\
M_{43} & M_{44}
\end{array}\right] ; & C_{r r}=\left[\begin{array}{ll}
C_{11} & C_{12} \\
C_{21} & C_{22}
\end{array}\right] ; \\
C_{r f}=\left[\begin{array}{ll}
C_{13} & C_{14} \\
C_{23} & C_{24}
\end{array}\right] ; & C_{f f}=\left[\begin{array}{ll}
C_{33} & C_{34} \\
C_{43} & C_{44}
\end{array}\right] ; \\
D_{f}=\left[\begin{array}{cc}
D_{f 1} & 0 \\
0 & D_{f 2}
\end{array}\right] ; & K_{f}=\left[\begin{array}{cc}
K_{f 1} & 0 \\
0 & K_{f 2}
\end{array}\right] .
\end{array}
$$

To develop a control law, the dynamical model (17) is written as two interconnected subsystems. Each subsystem has a pair of joint and link. There exists a nonsingular matrix of transformation $T_{r}$ such as

$$
\bar{q}=T_{r} q
$$

where $q=\left[\begin{array}{ll}q_{r} & q_{f}\end{array}\right]^{T}=\left[\begin{array}{llll}q_{r 1} & q_{r 2} & q_{f 1} & q_{f 2}\end{array}\right]^{T}$ is the original generalized coordinate, $\bar{q}=\left[\begin{array}{ll}q_{1} & q_{2}\end{array}\right]^{T}=\left[\begin{array}{llll}q_{r 1} & q_{r 2} & q_{f 1} & q_{f 2}\end{array}\right]^{T}$ is the transformed one, and the matrix of transformation $T_{r}$ is given by

$$
T_{r}=\left[\begin{array}{llll}
1 & 0 & 0 & 0 \\
0 & 0 & 1 & 0 \\
0 & 1 & 0 & 0 \\
0 & 0 & 0 & 1
\end{array}\right]
$$

Using the previous transformation, the dynamical model (17) can be written as follows:

$$
M(q) T_{r}^{-1} \ddot{\bar{q}}+C(q, \dot{q}) T_{r}^{-1} \dot{\bar{q}}+D T_{r}^{-1} \dot{\bar{q}}+K T_{r}^{-1} \bar{q}=L \tau,
$$

where $\ddot{\bar{q}}=T_{r} \ddot{q}$ and $\dot{\bar{q}}=T_{r} \dot{q}$.

Equation (23) is equivalent to the following expression:

$$
\bar{M}(q) \ddot{\bar{q}}+\bar{C}(q, \dot{q}) \dot{\bar{q}}+\bar{D} \dot{\bar{q}}+\bar{K} \bar{q}=\bar{L} \tau,
$$

where $\bar{M}=T_{r} M(q) T_{r}^{-1} ; \bar{C}=T_{r} C(q, \dot{q}) T_{r}^{-1} ; \bar{D}=T_{r} D T_{r}^{-1}$; $\bar{K}=T_{r} K T_{r}^{-1} ; \quad \bar{q}=\left[\begin{array}{ll}q_{1} & q_{2}\end{array}\right]^{T}=\left[\begin{array}{llll}q_{r 1} & q_{f 1} & q_{r 2} & q_{f 2}\end{array}\right]^{T} ; \bar{L}=$ $T_{r} L=\left[\begin{array}{llll}1 & 0 & 0 & 0 \\ 0 & 0 & 1 & 0\end{array}\right]^{T}$ and $\tau=\left[\begin{array}{l}\tau_{1} \\ \tau_{2}\end{array}\right]$.

The modified dynamical model (24) can be written as follows:

$$
\begin{aligned}
& {\left[\begin{array}{ll}
\bar{M}_{11} & \bar{M}_{12} \\
\bar{M}_{21} & \bar{M}_{22}
\end{array}\right]\left[\begin{array}{l}
\ddot{q}_{1} \\
\ddot{q}_{2}
\end{array}\right]+\left[\begin{array}{ll}
\bar{C}_{11} & \bar{C}_{12} \\
\bar{C}_{21} & \bar{C}_{22}
\end{array}\right]\left[\begin{array}{l}
\dot{q}_{1} \\
\dot{q}_{2}
\end{array}\right]} \\
& +\left[\begin{array}{ll}
\bar{D}_{11} & \bar{D}_{12} \\
\bar{D}_{21} & \bar{D}_{22}
\end{array}\right]\left[\begin{array}{l}
\dot{q}_{1} \\
\dot{q}_{2}
\end{array}\right]+\left[\begin{array}{ll}
\bar{K}_{11} & \bar{K}_{12} \\
\bar{K}_{21} & \bar{K}_{22}
\end{array}\right]\left[\begin{array}{l}
q_{1} \\
q_{2}
\end{array}\right]=\left[\begin{array}{l}
\bar{L}_{1} \tau \\
\bar{L}_{2} \tau
\end{array}\right],
\end{aligned}
$$

where

$$
\begin{aligned}
& \bar{M}_{11}=\left[\begin{array}{ll}
M_{11} & M_{13} \\
M_{31} & M_{33}
\end{array}\right] ; \quad \bar{M}_{12}=\left[\begin{array}{ll}
M_{12} & M_{14} \\
M_{32} & M_{34}
\end{array}\right] ; \\
& \bar{C}_{11}=\left[\begin{array}{ll}
C_{11} & C_{13} \\
C_{31} & C_{33}
\end{array}\right] ; \quad \bar{C}_{12}=\left[\begin{array}{ll}
C_{12} & C_{14} \\
C_{32} & C_{34}
\end{array}\right] ; \\
& \bar{D}_{11}=\left[\begin{array}{cc}
0 & 0 \\
0 & D_{f 1}
\end{array}\right] ; \quad \bar{D}_{12}=\left[\begin{array}{cc}
0 & 0 \\
0 & 0
\end{array}\right] \text {; } \\
& \bar{K}_{11}=\left[\begin{array}{cc}
0 & 0 \\
0 & K_{f 1}
\end{array}\right] ; \quad \bar{K}_{12}=\left[\begin{array}{cc}
0 & 0 \\
0 & 0
\end{array}\right] \text {; } \\
& \bar{M}_{21}=\left[\begin{array}{ll}
M_{21} & M_{23} \\
M_{41} & M_{43}
\end{array}\right] ; \quad \bar{M}_{22}=\left[\begin{array}{ll}
M_{22} & M_{24} \\
M_{42} & M_{44}
\end{array}\right] \text {; } \\
& \bar{C}_{21}=\left[\begin{array}{ll}
C_{21} & C_{23} \\
C_{41} & C_{43}
\end{array}\right] ; \quad \bar{C}_{22}=\left[\begin{array}{ll}
C_{22} & C_{24} \\
C_{42} & C_{44}
\end{array}\right] \text {; } \\
& \bar{D}_{21}=\left[\begin{array}{cc}
0 & 0 \\
0 & 0
\end{array}\right] ; \quad \bar{D}_{22}=\left[\begin{array}{cc}
0 & 0 \\
0 & D_{f 2}
\end{array}\right] \text {; } \\
& \bar{K}_{21}=\left[\begin{array}{cc}
0 & 0 \\
0 & 0
\end{array}\right] ; \quad \bar{K}_{22}=\left[\begin{array}{cc}
0 & 0 \\
0 & K_{f 2}
\end{array}\right] \text {; } \\
& q_{1}=\left[\begin{array}{c}
q_{r 1} \\
q_{f 1}
\end{array}\right] ; \quad q_{2}=\left[\begin{array}{c}
q_{r 2} \\
q_{f 2}
\end{array}\right] ; \\
& \bar{L}_{1}=\left[\begin{array}{ll}
1 & 0 \\
0 & 0
\end{array}\right] ; \quad \bar{L}_{2}=\left[\begin{array}{ll}
0 & 1 \\
0 & 0
\end{array}\right] .
\end{aligned}
$$

Equation (25) can be written in the following form that will be used for control law:

$$
\left[\begin{array}{c}
\bar{M}_{1}^{T} \\
\bar{M}_{2}^{T}
\end{array}\right] \ddot{\bar{q}}+\left[\begin{array}{c}
\bar{C}_{1}^{T} \\
\bar{C}_{2}^{T}
\end{array}\right] \dot{\bar{q}}+\left[\begin{array}{c}
\bar{D}_{1}^{T} \\
\bar{D}_{2}^{T}
\end{array}\right] \dot{\bar{q}}+\left[\begin{array}{c}
\bar{K}_{1}^{T} \\
\bar{K}_{2}^{T}
\end{array}\right] \bar{q}=\left[\begin{array}{l}
\bar{L}_{1} \\
\bar{L}_{2}
\end{array}\right] \tau,
$$

where $\bar{M}_{1}^{T}=\left[\bar{M}_{11} \bar{M}_{12}\right] ; \bar{C}_{1}^{T}=\left[\begin{array}{ll}\bar{C}_{11} & \bar{C}_{12}\end{array}\right] ; \bar{D}_{1}^{T}=$ $\left[\begin{array}{ll}\bar{D}_{11} & \bar{D}_{12}\end{array}\right] ; \bar{K}_{1}^{T}=\left[\begin{array}{ll}\bar{K}_{11} & \bar{K}_{12}\end{array}\right] ; \bar{M}_{2}^{T}=\left[\begin{array}{ll}\bar{M}_{21} & \bar{M}_{22}\end{array}\right] ; \bar{C}_{2}^{T}=$ $\left[\begin{array}{ll}\bar{C}_{21} & \bar{C}_{22}\end{array}\right] ; \bar{D}_{2}^{T}=\left[\begin{array}{ll}\bar{D}_{21} & \bar{D}_{22}\end{array}\right] ; \bar{K}_{2}^{T}=\left[\begin{array}{ll}\bar{K}_{21} & \bar{K}_{22}\end{array}\right]$.

The first and the second subsystems can be characterized by two mass and inertia symmetric positive definite matrices:

$$
\bar{M}_{11}=\left[\begin{array}{ll}
M_{11} & M_{13} \\
M_{31} & M_{33}
\end{array}\right], \quad \bar{M}_{22}=\left[\begin{array}{ll}
M_{22} & M_{24} \\
M_{42} & M_{44}
\end{array}\right] .
$$

The new generalized coordinate, used for the second joint and link while the first joint and link are assumed stable, is given as follows:

$$
\bar{Q}_{2}=\left[\begin{array}{ll}
q_{d 1} & q_{2}
\end{array}\right]^{T}=\left[\begin{array}{llll}
q_{r d 1} & q_{f d 1} & q_{r 2} & q_{f 2}
\end{array}\right]^{T} .
$$

Note that the coordinates of the first subsystem are the desired ones, and the coordinates of the second subsystem are the controlled ones. Using (27), the equation of motion of the second joint and link can be written as

$$
\bar{M}_{2}^{T}\left(\bar{Q}_{2}\right) \ddot{\bar{Q}}_{2}+\bar{C}_{2}^{T}\left(\bar{Q}_{2}, \dot{\bar{Q}}_{2}\right) \dot{\bar{Q}}_{2}+\bar{D}_{2} \dot{\bar{Q}}_{2}+\bar{K}_{2} \bar{Q}_{2}=\bar{L}_{2} \tau_{2} .
$$


The velocity $\dot{\bar{Q}}_{2}$ is the time derivative of $\bar{Q}_{2}$ and the acceleration $\ddot{\bar{Q}}_{2}$ is the time derivative of $\dot{\bar{Q}}_{2}$.

According to (8), the new noncollocated output is given as follows:

$$
y_{2}=q_{r 2}+\alpha_{2} \beta_{2}^{T} q_{f 2}, \quad 0 \leq \alpha_{2}<1,
$$

where $\beta_{2}^{T}=\beta_{2}=\emptyset_{21}\left(L_{2}\right) / L_{2}$ when considering just one mode. $\alpha_{2}$ is the weighted parameter characterizing the second noncollocated output.

Using (31), the equation of motion of the second subsystem is given as follows:

$$
\begin{aligned}
& M_{21} \ddot{q}_{r d 1}+M_{22} \ddot{y}_{2}+M_{23} \ddot{q}_{f d 1}+\left(M_{24}-\alpha_{2} \beta_{2} M_{22}\right) \ddot{q}_{f 2} \\
& +C_{21} \dot{q}_{r d 1}+C_{22} \dot{y}_{2}+C_{23} \dot{q}_{f d 1}+\mathbb{C}_{24} \dot{q}_{f 2}=\tau_{2}, \\
& M_{41} \ddot{q}_{r d 1}+M_{42} \ddot{y}_{2}+M_{43} \ddot{q}_{f d 1}+\left(M_{44}-\alpha_{2} \beta_{2} M_{42}\right) \ddot{q}_{f 2} \\
& \quad+C_{41} \dot{q}_{r d 1}+C_{42} \dot{y}_{2}+C_{43} \dot{q}_{f d 1}+\mathbb{C}_{44} \dot{q}_{f 2} \\
& +D_{f 2} \dot{q}_{f 2}+K_{f 2} q_{f 2}=0
\end{aligned}
$$

where $\mathbb{C}_{24}=C_{24}-\alpha_{2} \beta_{2} C_{22}$ and $\mathbb{C}_{44}=C_{44}-\alpha_{2} \beta_{2} C_{42}$.

The internal dynamics of the second link is deduced from (33) as follows:

$$
\begin{aligned}
\ddot{q}_{f 2}= & -\mathscr{M}_{41} \ddot{q}_{r d 1}-\mathscr{M}_{43} \ddot{q}_{f d 1}-\mathscr{M}_{42} \ddot{y}_{2}-\mathscr{C}_{41} \dot{q}_{r d 1} \\
& -\mathscr{C}_{42} \dot{y}_{2}-\mathscr{C}_{43} \dot{q}_{f d 1}-\mathscr{C}_{44} \dot{q}_{f 2}-\mathscr{K}_{f 2} q_{f 2}-\mathscr{D}_{f 2} \dot{q}_{f 2},
\end{aligned}
$$

where $\mathscr{M}_{41}=\left(M_{44}-\alpha_{2} \beta_{2} M_{42}\right)^{-1} M_{41} ; \mathscr{M}_{42}=\left(M_{44}-\right.$ $\left.\alpha_{2} \beta_{2} M_{42}\right)^{-1} M_{42} ; \mathscr{M}_{43}=\left(M_{44}-\alpha_{2} \beta_{2} M_{42}\right)^{-1} M_{43} ; \mathscr{C}_{41}=$ $\left(M_{44}-\alpha_{2} \beta_{2} M_{42}\right)^{-1} C_{41} ; \mathscr{C}_{42}=\left(M_{44}-\alpha_{2} \beta_{2} M_{42}\right)^{-1} C_{42}$; $\mathscr{C}_{44}=\left(M_{44}-\alpha_{2} \beta_{2} M_{42}\right)^{-1} \mathbb{C}_{44} ; \mathscr{K}_{f 2}=\left(M_{44}-\alpha_{2} \beta_{2} M_{42}\right)^{-1}$ $K_{f 2} ; \mathscr{D}_{f 2}=\left(M_{44}-\alpha_{2} \beta_{2} M_{42}\right)^{-1} D_{f 2}$, and $\alpha_{2}$ is chosen such as $\alpha_{2} \neq\left(M_{44} / \beta_{2} M_{42}\right)$.

Inserting (34) in (32), we get

$$
\begin{aligned}
& M_{21}^{*} \ddot{q}_{r d 1}+M_{22}^{*} \ddot{y}_{2}+M_{23}^{*} \ddot{q}_{f d 1}+C_{21}^{*} \dot{q}_{r d 1} \\
& \quad+C_{22}^{*} \dot{y}_{2}+C_{23}^{*} \dot{q}_{f d 1}+C_{24}^{*} \dot{q}_{f 2}+K_{f 2}^{*} q_{f 2}+D_{f 2}^{*} \dot{q}_{f 2}=\tau_{2},
\end{aligned}
$$

where $M_{21}^{*}=M_{21}-\left(M_{24}-\alpha_{2} \beta_{2} M_{22}\right) \mathscr{M}_{41} ; M_{22}^{*}=M_{22}-$ $\left(M_{24}-\alpha_{2} \beta_{2} M_{22}\right) \mathscr{M}_{42} ; M_{23}^{*}=M_{23}-\left(M_{24}-\alpha_{2} \beta_{2} M_{22}\right) \mathscr{M}_{43}$; $C_{21}^{*}=C_{21}-\left(M_{24}-\alpha_{2} \beta_{2} M_{22}\right) \mathscr{C}_{41} ; C_{22}^{*}=C_{22}-\left(M_{24}-\right.$ $\left.\alpha_{2} \beta_{2} M_{22}\right) \mathscr{C}_{42} ; C_{23}^{*}=C_{23}-\left(M_{24}-\alpha_{2} \beta_{2} M_{22}\right) \mathscr{C}_{43} ; C_{24}^{*}=$ $\bar{C}_{24}-\left(M_{24}-\alpha_{2} \beta_{2} M_{22}\right) \mathscr{C}_{44} ; K_{f 2}^{*}=-\left(M_{24}-\alpha_{2} \beta_{2} M_{22}\right) \mathscr{K}_{f 2}$ and $D_{f 2}^{*}=-\left(M_{24}-\alpha_{2} \beta_{2} M_{22}\right) \mathscr{D}_{f 2}$.

To control the second link, we propose the following control law:

$$
\tau_{2}=\tau_{r 2}+\tau_{f 2}+\delta \tau_{2}
$$

where $\tau_{r 2}$ is used for the rigid part, $\tau_{f 2}$ is for the flexible part, and $\delta \tau_{2}$ is the setting term:

$$
\begin{aligned}
\tau_{r 2}= & K_{p 2} \tilde{y}_{2}+K_{d 2} \dot{\tilde{y}}_{2}+M_{22}^{*} \ddot{y}_{2}^{*} \\
& +M_{21}^{*} \ddot{q}_{r d 1}+C_{21}^{*} \dot{q}_{r d 1}+C_{22}^{*} \dot{y}_{d 2}, \\
\tau_{f 2}= & C_{23}^{*} \dot{q}_{f d 1}+C_{24}^{*} \dot{q}_{f 2}+\alpha_{2} \beta_{2}\left(M_{21}^{*} \ddot{q}_{f d 1}+C_{21}^{*} \dot{q}_{f d 1}\right) \\
& +K_{f 2}^{*} q_{f 2}+D_{f 2}^{*} \dot{q}_{f 2}, \\
\delta \tau_{2}= & \delta M_{22}^{*} \ddot{y}_{2}^{*}+\delta M_{21}^{*} \ddot{y}_{d 1}+\delta C_{21}^{*} \dot{y}_{d 1} \\
& +\delta C_{22}^{*} \dot{y}_{d 2}+\delta C_{23}^{*} \dot{q}_{f d 1}+\delta C_{24}^{*} \dot{q}_{f 2},
\end{aligned}
$$

where $\tilde{y}_{2}=y_{d 2}-y_{2} ; \dot{\tilde{y}}_{2}=\dot{y}_{d 2}-\dot{y}_{2}$ and $\ddot{y}_{2}^{*}=\ddot{y}_{d 2}+\lambda_{2} \dot{\tilde{y}}_{2}$; $\lambda_{2}>0$.

Now going backward to the first subsystem and assuming that the second subsystem is stable, the new generalized coordinate associated to the second subsystem becomes

$$
\bar{Q}_{1}=\left[\begin{array}{ll}
q_{1} & q_{d 2}
\end{array}\right]^{T}=\left[\begin{array}{llll}
q_{r 1} & q_{f 1} & q_{r d 2} & q_{f d 2}
\end{array}\right]^{T} .
$$

Using (27), the equation of motion of the first subsystem (i.e., first link and joint) is given as follows:

$$
\bar{M}_{1}^{T}\left(\bar{Q}_{1}\right) \ddot{\bar{Q}}_{1}+\bar{C}_{1}^{T}\left(\bar{Q}_{1}, \dot{\bar{Q}}_{1}\right) \dot{\bar{Q}}_{1}+\bar{D}_{1} \dot{\bar{Q}}_{1}+\bar{K}_{1} \bar{Q}_{1}=\bar{L}_{1} \tau_{1} .
$$

The new noncollocated output, defined as the angle of the first motor augmented by a weighted value of the link's extremity angle, is given as follows:

$$
y_{1}=q_{r 1}+\alpha_{1} \beta_{1}^{T} q_{f 1}, \quad 0 \leq \alpha_{1}<1,
$$

where $\beta_{1}^{T}=\beta_{1}=\emptyset_{11}\left(L_{1}\right) / L_{1}$ for one mode.

Using (39) and (40), the equation of motion of the first joint and link is given as follows:

$$
\begin{aligned}
& M_{11} \ddot{y}_{1}+M_{12} \ddot{q}_{r d 2}+\left(M_{13}-\alpha_{1} \beta_{1} M_{11}\right) \ddot{q}_{f 1} \\
& +M_{14} \ddot{q}_{f d 2}+C_{11} \dot{y}_{1}+C_{12} \dot{q}_{r d 2} \\
& \quad+\mathbb{C}_{13} \dot{q}_{f 1}+C_{14} \dot{q}_{f d 2}=\tau_{1}, \\
& M_{31} \ddot{y}_{1}+M_{32} \ddot{q}_{r d 2}+\left(M_{33}-\alpha_{1} \beta_{1} M_{31}\right) \ddot{q}_{f 1} \\
& \quad+M_{34} \ddot{q}_{f d 2}+C_{31} \dot{y}_{1}+C_{32} \dot{q}_{r d 2}+\mathbb{C}_{33} \dot{q}_{f 1} \\
& +C_{34} \dot{q}_{f d 2}+D_{f 1} \dot{q}_{f 1}+K_{f 1} q_{f 1}=0 .
\end{aligned}
$$

Let us define $\mathbb{C}_{13}=C_{13}-\alpha_{1} \beta_{1} C_{11}$ and $\mathbb{C}_{33}=C_{33}-\alpha_{1} \beta_{1} C_{31}$.

To find the internal dynamics of the first link, $\ddot{q}_{f 1}$ is given from (42) as follows:

$$
\begin{aligned}
\ddot{q}_{f 1}= & -\mathscr{M}_{31} \ddot{y}_{1}-\mathscr{M}_{32} \ddot{q}_{r d 2}-\mathscr{M}_{34} \ddot{q}_{f d 2}-\mathscr{C}_{31} \dot{y}_{1}-\mathscr{C}_{32} \dot{q}_{r d 2} \\
& -\mathscr{C}_{33} \dot{q}_{f 1}-\mathscr{C}_{34} \dot{q}_{f d 2}-\mathscr{K}_{f 1} q_{f 1}-\mathscr{D}_{f 1} \dot{q}_{f 1},
\end{aligned}
$$


where $\mathscr{M}_{31}=\left(M_{33}-\alpha_{1} \beta_{1} M_{31}\right)^{-1} M_{31} ; \mathscr{M}_{32}=\left(M_{33}-\right.$ $\left.\alpha_{1} \beta_{1} M_{31}\right)^{-1} M_{32} ; \mathscr{M}_{34}=\left(M_{33}-\alpha_{1} \beta_{1} M_{31}\right)^{-1} M_{34} ; \mathscr{C}_{31}=$ $\left(M_{33}-\alpha_{1} \beta_{1} M_{31}\right)^{-1} C_{31} ; \mathscr{C}_{33}=\left(M_{33}-\alpha_{1} \beta_{1} M_{31}\right)^{-1} \mathbb{C}_{33} ;$ $\mathscr{C}_{34}=\left(M_{33}-\alpha_{1} \beta_{1} M_{31}\right)^{-1} C_{34} ; \mathscr{K}_{f 1}=\left(M_{33}-\alpha_{1} \beta_{1} M_{31}\right)^{-1}$ $K_{f 1} ; \mathscr{D}_{f 1}=\left(M_{33}-\alpha_{1} \beta_{1} M_{31}\right)^{-1} D_{f 1}$, and $\alpha_{1}$ is selected such as $\alpha_{1} \neq M_{33} / \beta_{1} M_{31}$. Using the same strategy, the control law of the first subsystem is given as follows:

$$
\tau_{1}=\tau_{r 1}+\tau_{f 1}+\delta \tau_{1}
$$

where

$$
\begin{aligned}
\tau_{r 1}= & K_{p 1} \tilde{y}_{1}+K_{d 1} \dot{\tilde{y}}_{1}+M_{11}^{*} \ddot{y}_{1}^{*} \\
& +M_{12}^{*} \ddot{q}_{r d 2}+C_{11}^{*} \dot{y}_{d 1}+C_{12}^{*} \dot{q}_{r d 2} ; \\
\tau_{f 1}= & C_{13}^{*} \dot{q}_{f 1}+C_{14}^{*} \dot{q}_{f d 2}+\alpha_{1} \beta_{1}\left(M_{12}^{*} \ddot{q}_{f d 2}+C_{12}^{*} \dot{q}_{f d 2}\right) \\
& +K_{f 1}^{*} q_{f 1}+D_{f 1}^{*} \dot{q}_{f 1} ; \\
\delta \tau_{1}= & \delta M_{11}^{*} \ddot{y}_{1}^{*}+\delta M_{12}^{*} \ddot{y}_{d 2}+\delta C_{11}^{*} \dot{y}_{d 1}+\delta C_{12}^{*} \dot{y}_{d 2} \\
& +\delta C_{13}^{*} \dot{q}_{f 1}+\delta C_{14}^{*} \dot{q}_{f d 2} ;
\end{aligned}
$$

where $\tilde{y}_{1}=y_{d 1}-y_{1} ; \ddot{y}_{2}^{*}=\ddot{y}_{d 2}+\lambda_{2} \dot{\tilde{y}}_{2}$ and $\dot{\tilde{y}}_{2}=\dot{y}_{d 2}-\dot{y}_{2}$; $\lambda_{2}>0 . M_{1 j}^{*}=M_{1 j}-\left(M_{13}-\alpha_{1} \beta_{1} M_{11}\right) M_{3 j}, j=1,2,4 ; C_{1 j}^{*}=$ $C_{1 j}-\left(M_{13}-\alpha_{1} \beta_{1} M_{11}\right) \mathscr{C}_{3 j}, j=1,2,3,4 ; K_{f 1}^{*}=-\left(M_{13}-\right.$ $\left.\alpha_{1} \beta_{1} M_{11}\right) \mathscr{K}_{f 1} ; D_{f 1}^{*}=-\left(M_{13}-\alpha_{1} \beta_{1} M_{11}\right) \mathscr{D}_{f 1}$. The distributed control strategy can be represented by Figure 3

\section{Stability Analysis}

This section presents the stability analysis of the tracking errors of the rigid and the flexible parts. First, we study the global stability of the rigid part by inserting the two control laws in the initial dynamical model. Second, the stability of the flexible part is studied to select the weighted parameters and guarantee bounded internal dynamics such that the new output is as close as possible to the tip.

4.1. Stability of Rigid Part. In compact form, the first (36) and second (44) control laws can be written as follows:

$$
\tau=\tau_{r}+\tau_{f}+\delta \tau,
$$

where

$$
\begin{gathered}
\tau_{r}=K_{p} \tilde{y}+K_{d} \dot{\tilde{y}}+M_{r d}^{*} \ddot{y}^{*}+M_{r n d}^{*} \ddot{q}_{r d}+C_{r d}^{*} \dot{y}_{d}+C_{r n d}^{*} \dot{q}_{r d} ; \\
\tau_{f}=C_{r f}^{*} \dot{q}_{f}+\alpha \beta\left(M_{r n d}^{*} \ddot{q}_{f d}+C_{r n d}^{*} \dot{q}_{f d}\right)+K_{f}^{*} q_{f}+D_{f}^{*} \dot{q}_{f} ; \\
\delta \tau=\delta M_{r d}^{*} \ddot{y}^{*}+\delta M_{r n d}^{*} \ddot{y}_{d}+\delta C_{r}^{*} \dot{y}_{d}+\delta C_{r f}^{*} \dot{q}_{f} ;
\end{gathered}
$$

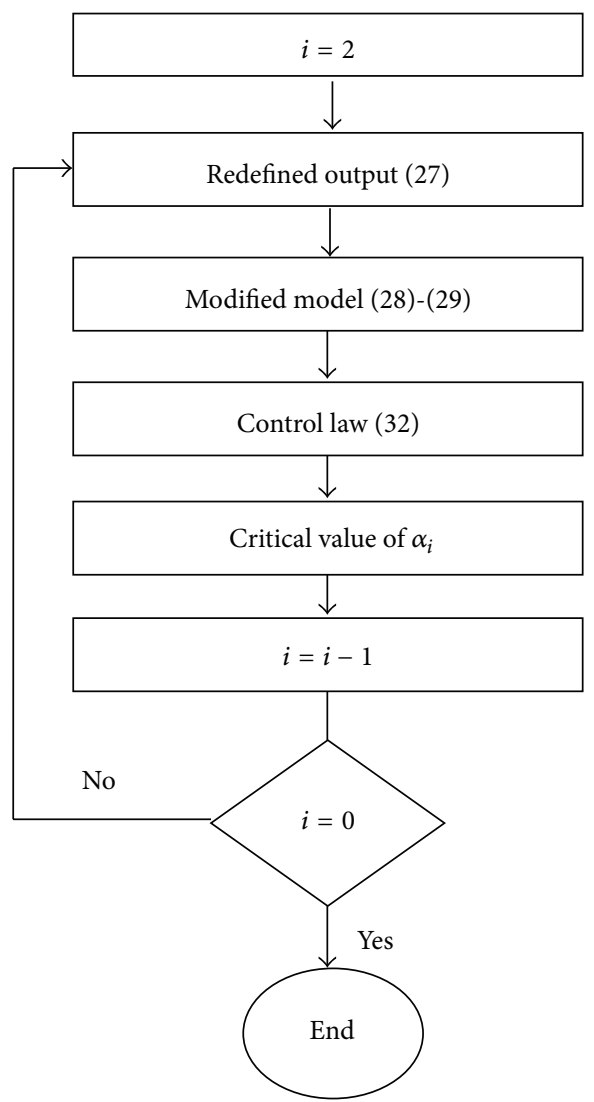

FIGURE 3: Distributed control strategy.

$$
\begin{array}{cc}
K_{p}=\left[\begin{array}{cc}
K_{p 1} & 0 \\
0 & K_{p 2}
\end{array}\right] ; & K_{d}=\left[\begin{array}{cc}
K_{d 1} & 0 \\
0 & K_{d 2}
\end{array}\right] ; \\
M_{r d}^{*}=\left[\begin{array}{cc}
M_{11}^{*} & 0 \\
0 & M_{22}^{*}
\end{array}\right] ; & M_{r n d}^{*}=\left[\begin{array}{cc}
0 & M_{12}^{*} \\
M_{21}^{*} & 0
\end{array}\right] ; \\
C_{r d}^{*}=\left[\begin{array}{cc}
C_{11}^{*} & 0 \\
0 & C_{22}^{*}
\end{array}\right] ; & C_{r n d}^{*}=\left[\begin{array}{cc}
0 & C_{12}^{*} \\
C_{21}^{*} & 0
\end{array}\right] ; \\
C_{r f}^{*}=\left[\begin{array}{cc}
C_{13}^{*} & C_{14}^{*} \\
C_{23}^{*} & C_{24}^{*}
\end{array}\right] ; & \delta M_{r d}^{*}=\left[\begin{array}{cc}
\delta M_{11}^{*} & 0 \\
0 & \delta M_{22}^{*}
\end{array}\right] ; \\
\delta M_{r n d}^{*}=\left[\begin{array}{cc}
0 & \delta M_{12}^{*} \\
\delta M_{21}^{*} & 0
\end{array}\right] ; & \delta C_{r}^{*}=\left[\begin{array}{ll}
\delta C_{11}^{*} & \delta C_{12}^{*} \\
\delta C_{21}^{*} & \delta C_{22}^{*}
\end{array}\right] ; \\
\delta C_{r f}^{*}=\left[\begin{array}{ll}
\delta C_{13}^{*} & \delta C_{14}^{*} \\
\delta C_{23}^{*} & \delta C_{24}^{*}
\end{array}\right] ;
\end{array}
$$

$\delta M_{i j}^{*}$ are given in (B.3).

To study the global stability, we insert the control law given in (46) in the dynamical model given in (1).

Proposition 1. The equation of motion of the two-flexible-link manipulator (17) is equivalent to the following model:

$$
\begin{aligned}
& M_{r}^{*}\left(q_{\alpha}\right) \ddot{y}+C_{r}^{*}\left(q_{\alpha}, \dot{q}_{\alpha}\right) \dot{y}+C_{r f}^{*}\left(q_{\alpha}, \dot{q}_{\alpha}\right) \dot{q}_{f} \\
& +K_{f}^{*} q_{f}+D_{f}^{*} \dot{q}_{f}=\tau,
\end{aligned}
$$


where $q_{\alpha}=\left[\begin{array}{llll}y_{1} & y_{2} & q_{f 1} & q_{f 2}\end{array}\right]^{T} ; M_{r}^{*}=\left[\begin{array}{ll}M_{11}^{*} & M_{12}^{*} \\ M_{21}^{*} & M_{22}^{*}\end{array}\right]$ is positive definite matrix; $C_{r}^{*}=\left[\begin{array}{ll}C_{11}^{*} & C_{12}^{*} \\ C_{21}^{*} & C_{22}^{*}\end{array}\right]$ and $C_{r f}^{*}=\left[\begin{array}{ll}C_{13}^{*} & C_{14}^{*} \\ C_{23}^{*} & C_{24}^{*}\end{array}\right] ; M_{r}^{*}$ and $C_{r}^{*}$ satisfy the following skew-symmetric property:

$$
X^{T}\left(\dot{M}_{r}^{*}-2 C_{r}^{*}\right) X=0, \quad \forall X \in \mathscr{R}^{n} .
$$

Proof. See Appendix A.

$M_{r}^{*}$ can be written as

$$
M_{r}^{*}=M_{r d}^{*}+M_{r n d}^{*}
$$

where $M_{r d}^{*}$ is the diagonal elements and $M_{r n d}^{*}$ is the nondiagonal elements of $M_{r d}^{*}$. Then, the dynamical model (50) can be written as follows:

$$
\begin{aligned}
& M_{r d}^{*}\left(q_{\alpha}\right) \ddot{y}+M_{r n d}^{*}\left(q_{\alpha}\right) \ddot{y}+C_{r}^{*}\left(q_{\alpha}, \dot{q}_{\alpha}\right) \dot{y} \\
& \quad+C_{r f}^{*}\left(q_{\alpha}, \dot{q}_{\alpha}\right) \dot{q}_{f}+K_{f}^{*} q_{f}+D_{f}^{*} \dot{q}_{f}=\tau .
\end{aligned}
$$

Using (52), the control law (46) is equivalent to

$$
\begin{aligned}
\tau= & K_{p} \tilde{y}+K_{d} \dot{\tilde{y}}+M_{r d}^{*}(Q) \ddot{y}^{*}+M_{r n d}^{*}(Q) \ddot{y}_{d}+C_{r}^{*}(Q, \dot{Q}) \dot{y}_{d} \\
& +C_{r f}^{*}(Q, \dot{Q}) \dot{q}_{f}+K_{f}^{*} q_{f}+D_{f}^{*} \dot{q}_{f}+\delta \tau .
\end{aligned}
$$

Now, the global stability is studied by inserting the control law (54) in the dynamical model (53). Then, the error dynamics are given by the following equation:

$$
\begin{aligned}
K_{p} \tilde{y} & +K_{d} \dot{\tilde{y}}+\left[M_{r d}^{*}(Q) \ddot{y}^{*}-M_{r d}^{*}\left(q_{\alpha}\right) \ddot{y}\right] \\
& +\left[M_{r n d}^{*}(Q) \ddot{y}_{d}-M_{r n d}^{*}\left(q_{\alpha}\right) \ddot{y}\right] \\
& +\left[C_{r}^{*}(Q, \dot{Q}) \dot{y}_{d}-C_{r}^{*}\left(q_{\alpha}, \dot{q}_{\alpha}\right) \dot{y}\right] \\
& +\left[C_{r f}^{*}(Q, \dot{Q}) \dot{q}_{f}-C_{r f}^{*}\left(q_{\alpha}, \dot{q}_{\alpha}\right) \dot{q}_{f}\right]+\delta \tau=0 .
\end{aligned}
$$

Proposition 2. When using the control law (54) and the dynamical model (53), the error dynamics given by (55) are equivalent to

$$
K_{p} \tilde{y}+K_{d} \dot{\tilde{y}}+M_{r}^{*}\left(q_{\alpha}\right) \ddot{\tilde{y}}+C_{r}^{*}\left(q_{\alpha}, \dot{q}_{\alpha}\right) \dot{\tilde{y}}+\lambda M_{r d}^{*}\left(q_{\alpha}\right) \dot{\tilde{y}}=0 .
$$

\section{Proof. See Appendix B.}

To prove the asymptotical stability of the error dynamics, we propose the following Lyapunov function:

$$
V=\frac{1}{2} \dot{\tilde{y}}^{T} M_{r}^{*} \dot{\tilde{y}}+\frac{1}{2} \tilde{y}^{T} K_{d} \tilde{y}
$$

The time derivative of $V$ is

$$
\begin{aligned}
\dot{V}(t)= & \dot{\tilde{y}}^{T} M_{r}^{*} \ddot{\tilde{y}}+\frac{1}{2} \dot{\tilde{y}}^{T} \dot{M}_{r}^{*} \dot{\tilde{y}}+\tilde{y}^{T} K_{p} \dot{\tilde{y}} \\
= & \dot{\tilde{y}}^{T}\left(-C_{r}^{*} \dot{\tilde{y}}-\lambda M_{r d}^{*} \dot{\tilde{y}}-K_{p} \tilde{y}-K_{d} \dot{\tilde{y}}\right) \\
& +\frac{1}{2} \dot{\tilde{y}}^{T} \dot{M}_{r}^{*} \dot{\tilde{y}}+\tilde{y}^{T} K_{p} \dot{\tilde{y}} .
\end{aligned}
$$

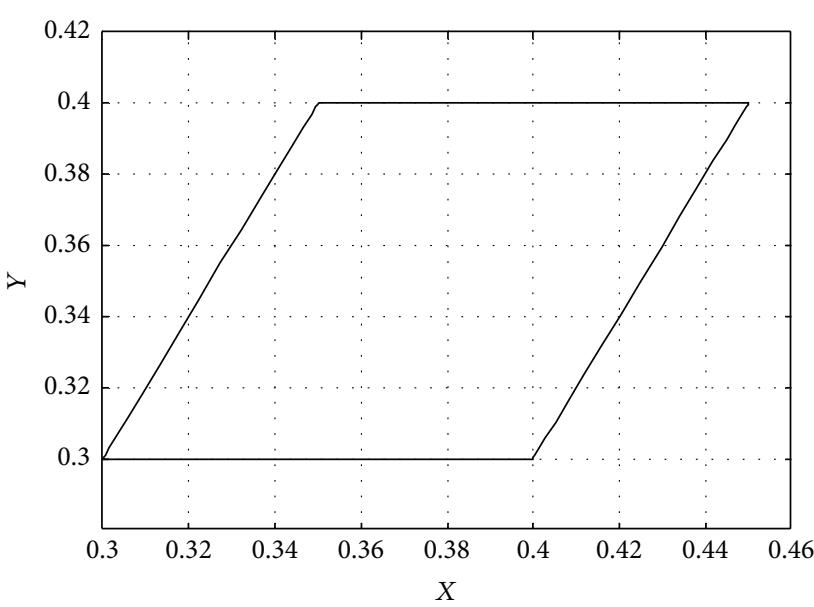

FIGURE 4: $X-Y$ desired workspace trajectory.

Using Proposition 1, we can conclude that

$$
\dot{V}(t)=-\dot{\tilde{y}}^{T}\left(K_{d}+\lambda M_{r d}^{*}\right) \dot{\tilde{y}}
$$

$K_{d}, \lambda$, and $M_{r d}^{*}$ are positive definite diagonal matrices. Then, using LaSalle theorem [24], the error dynamics are globally asymptotically stable.

4.2. Stability of the Flexible Part. In this section, asymptotical stability of the flexible part is studied using the internal dynamics. From the dynamical model given in (1) and (3), the equation of motion of the flexible part of the second link is given by

$$
\begin{gathered}
M_{41} \ddot{q}_{r 1}+M_{42} \ddot{q}_{r 2}+M_{43} \ddot{q}_{f 1}+M_{44} \ddot{q}_{f 2} \\
+H_{4}+D_{f 2} \dot{q}_{f 2}+K_{f 2} q_{f 2}=0 .
\end{gathered}
$$

Using the new generalized coordinate (29) and the new noncollocated output (31), the equation (60) is equivalent to

$$
\begin{aligned}
& M_{41} \ddot{q}_{r d 1}+M_{42} \ddot{y}_{2}+M_{43} \ddot{q}_{f d 1}+\left(M_{44}-\alpha_{2} \beta_{2} M_{42}\right) \ddot{q}_{f 2} \\
& +H_{4}+D_{f 2} \dot{q}_{f 2}+K_{f 2} q_{f 2}=0 .
\end{aligned}
$$

The internal dynamics of the second link are given by subtracting $\ddot{q}_{f 2}$ from (61) as follows:

$$
\begin{aligned}
\ddot{q}_{f 2}= & -\mathscr{M}_{41} \ddot{q}_{r d 1}-\mathscr{M}_{42} \ddot{y}_{2}-\mathscr{M}_{43} \ddot{q}_{f d 1} \\
& -\mathscr{H}_{4}-\mathscr{K}_{f 2} q_{f 2}-\mathscr{D}_{f 2} \dot{q}_{f 2},
\end{aligned}
$$

where $\mathscr{M}_{41}=\left(M_{44}-\alpha_{2} \beta_{2} M_{42}\right)^{-1} M_{41} ; \mathscr{M}_{42}=\left(M_{44}{ }^{-}\right.$ $\left.\alpha_{2} \beta_{2} M_{42}\right)^{-1} M_{42} ; \mathscr{M}_{43}=\left(M_{44}-\alpha_{2} \beta_{2} M_{42}\right)^{-1} M_{43} ; \mathscr{H}_{4}=$ $\left(M_{44}-\alpha_{2} \beta_{2} M_{42}\right)^{-1} H_{4} ; \mathscr{D}_{f 2}=\left(M_{44}-\alpha_{2} \beta_{2} M_{42}\right)^{-1} D_{f 2}$; $\mathscr{K}_{f 2}=\left(M_{44}-\alpha_{2} \beta_{2} M_{42}\right)^{-1} K_{f 2}$, and $\alpha_{2}$ is chosen such as $\alpha_{2} \neq M_{44} / \beta_{2} M_{42}$.

The quasi-static approach, using the inverse dynamics, neglects $\ddot{q}_{f d 1}$. Then, the tracking errors of the rigid and 


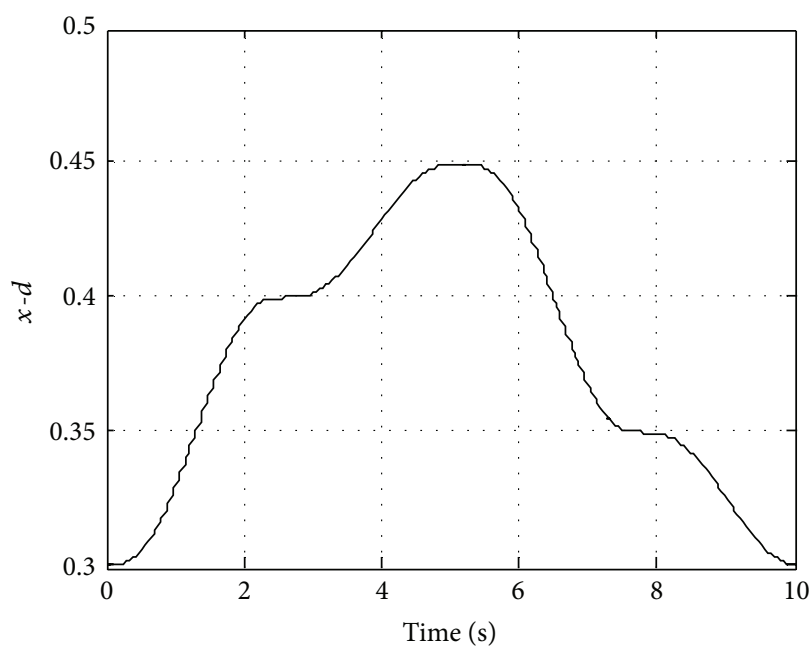

(a)

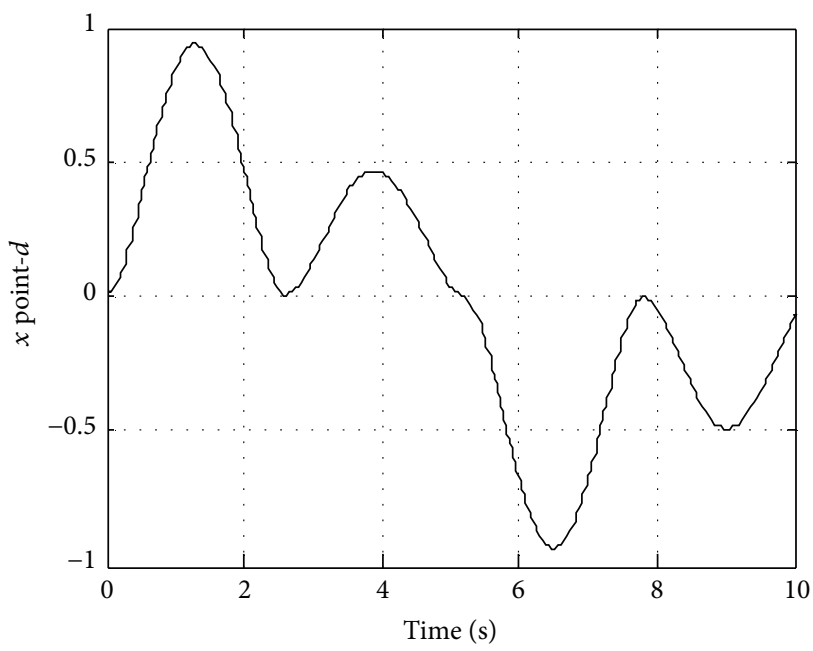

(c)

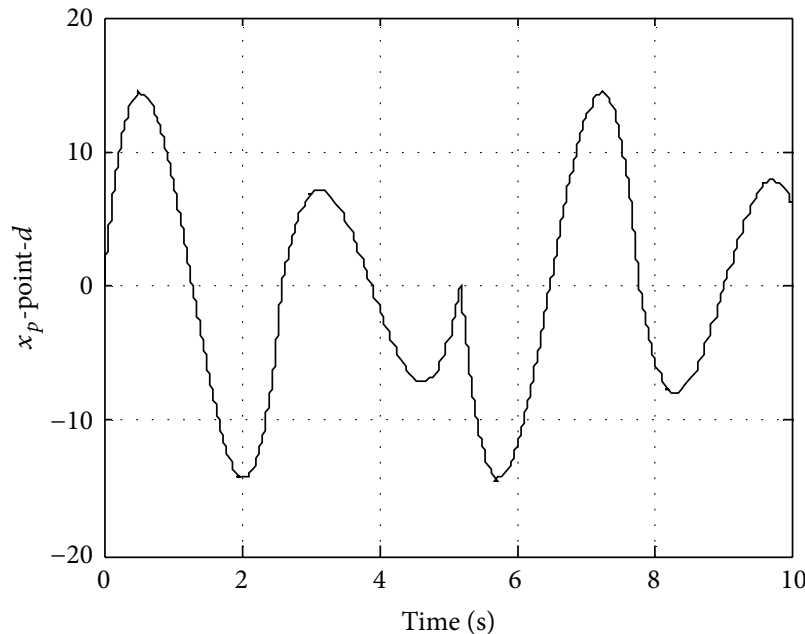

(e)

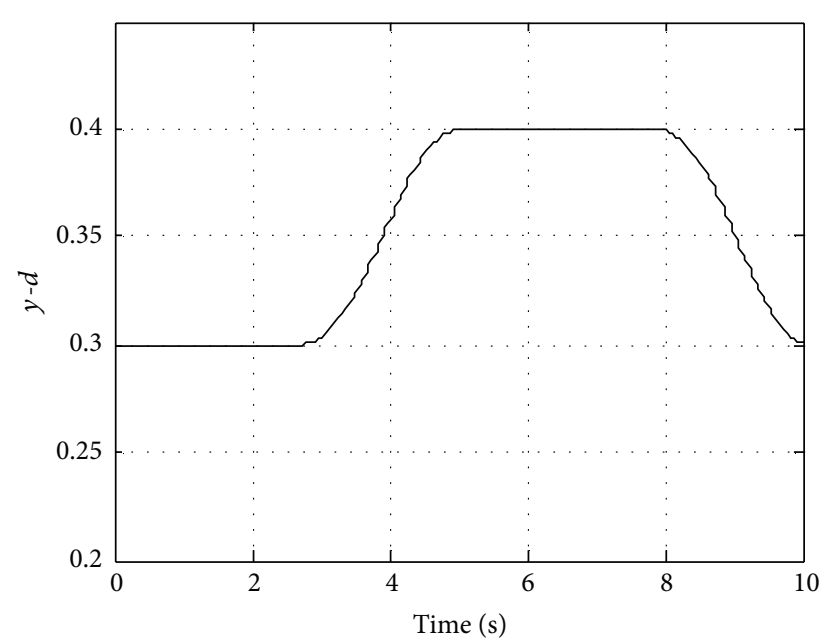

(b)

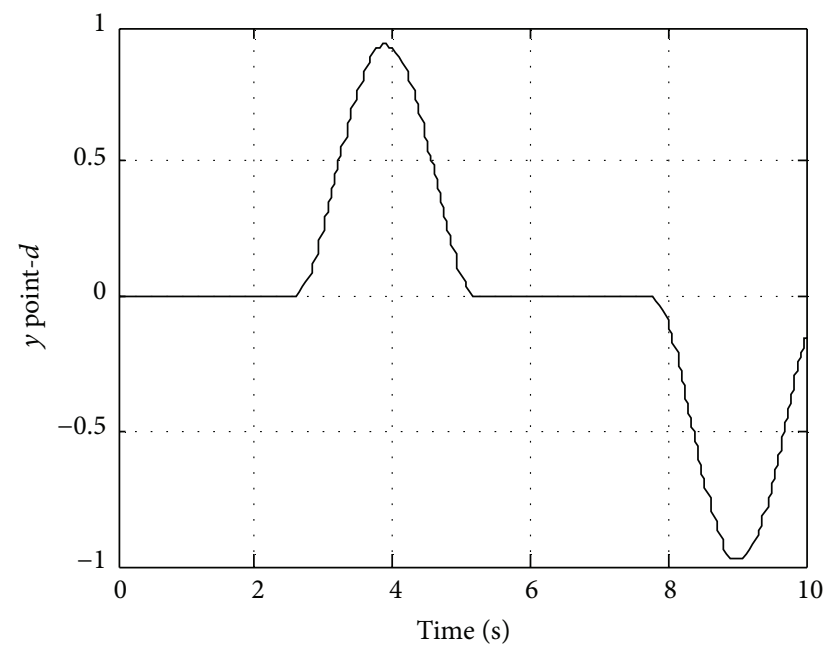

(d)

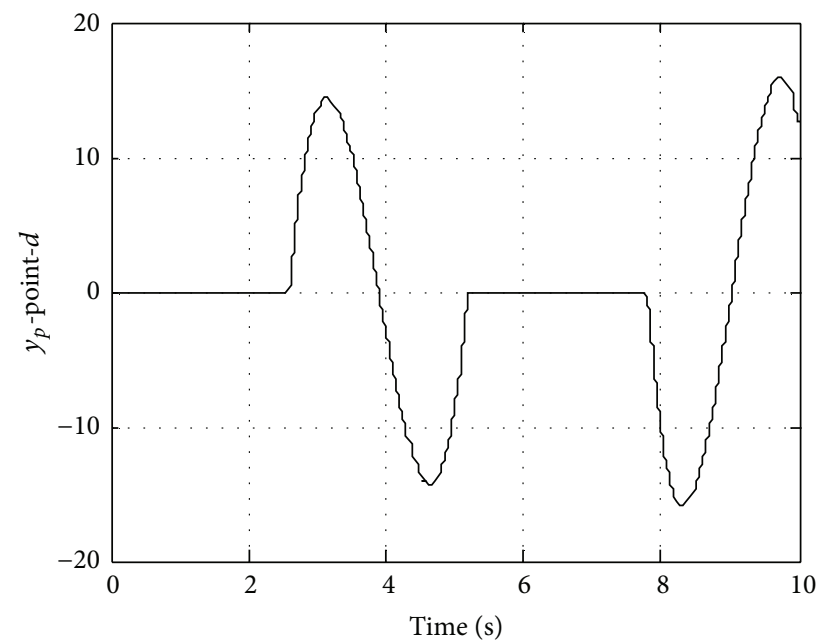

(f)

FIGURE 5: Desired workspace trajectory: (a)-(b) position trajectory, (c)-(d) velocity trajectories, and (e)-(f) acceleration trajectories. 


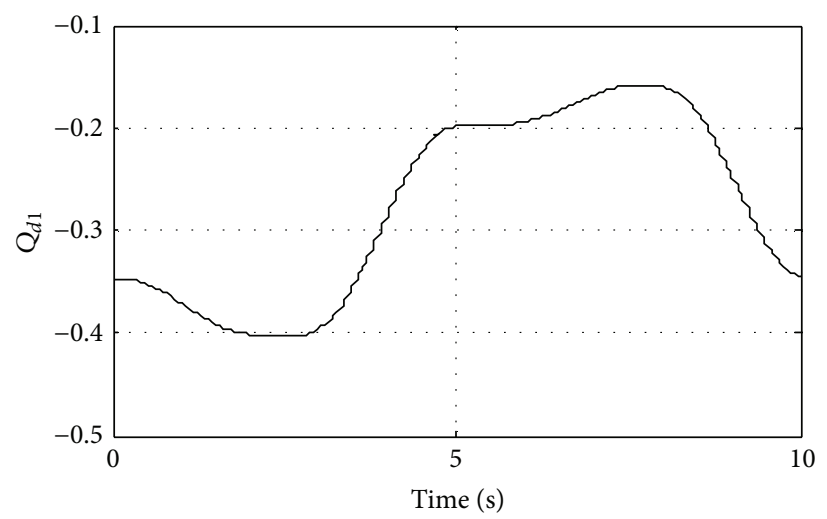

(a)

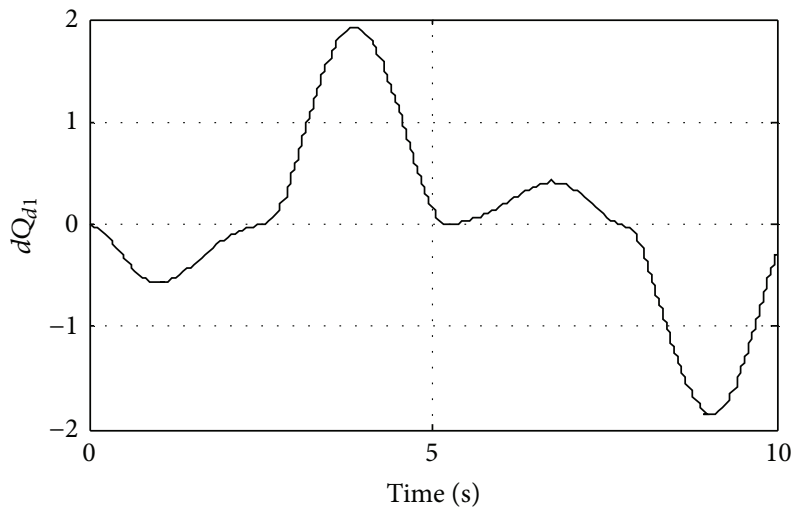

(c)

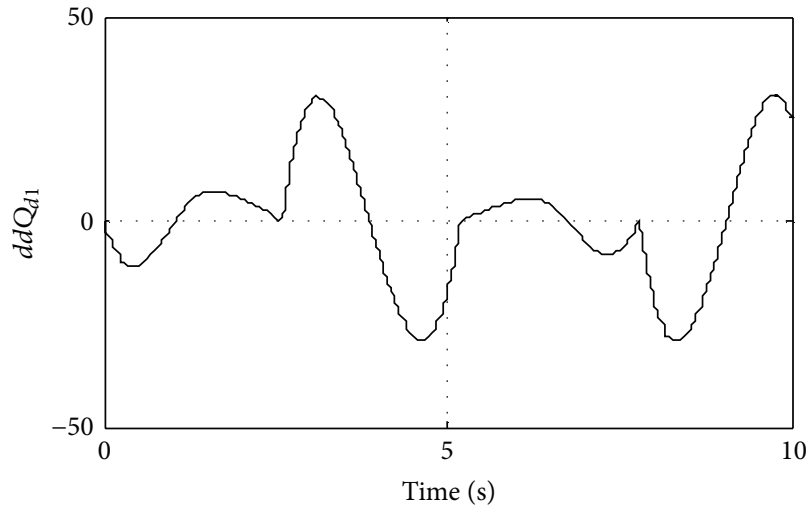

(e)

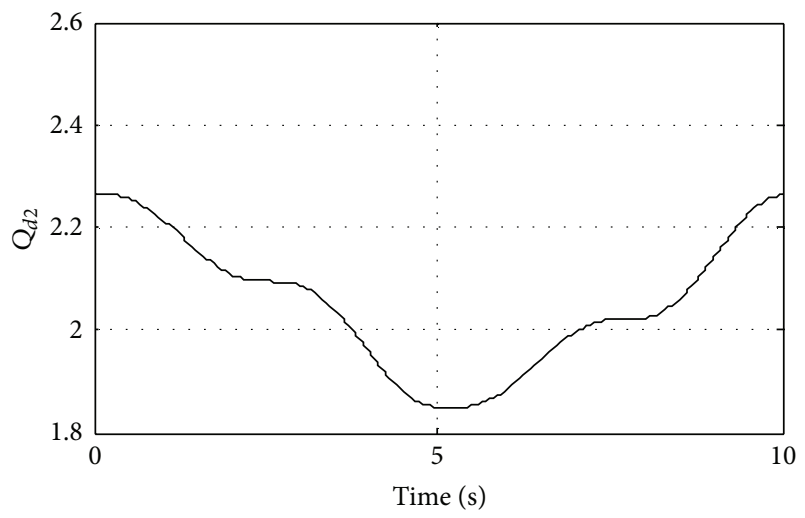

(b)

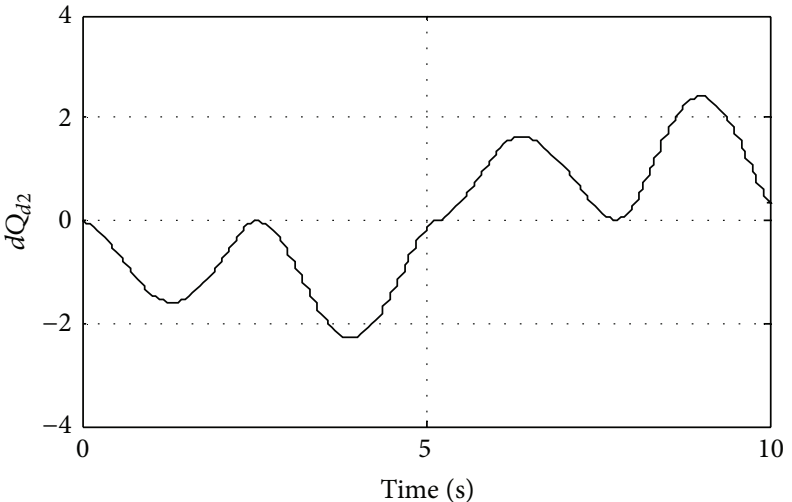

(d)

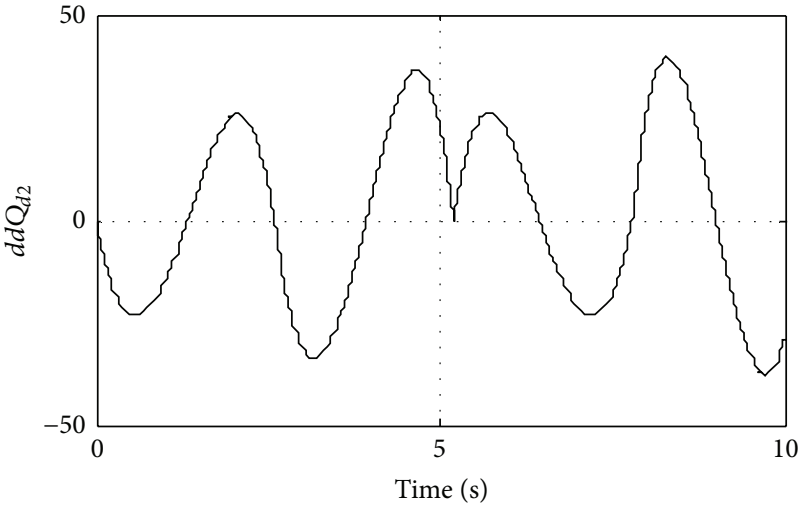

(f)

Figure 6: Desired virtual space trajectories: (a)-(b) position trajectories, (c)-(d) velocity trajectories, and (e)-(f) acceleration trajectories.

flexible part of the second link are given by the following expressions:

$$
\begin{aligned}
\ddot{\tilde{q}}_{f 2}= & \ddot{q}_{f d 2}-\ddot{q}_{f 2}=-\left(\mathscr{M}_{d 41}-\mathscr{M}_{41}\right) \ddot{q}_{r d 1} \\
& -\mathscr{M}_{d 42} \ddot{\tilde{y}}_{2}-\left(\mathscr{H}_{d 4}-\mathscr{H}_{4}\right) \\
& -\left(\mathscr{K}_{f d 2} q_{f d 2}-\mathscr{K}_{f 2} q_{f 2}\right) \\
& -\left(\mathscr{D}_{f d 2} \dot{q}_{f d 2}-\mathscr{D}_{f 2} \dot{q}_{f 2}\right) .
\end{aligned}
$$

In the above section, the tracking error of the rigid part was proved to be asymptotically stable; then, $\tilde{y}_{2}, \dot{\tilde{y}}_{2}, \ddot{\tilde{y}}_{2} \rightarrow 0$ as $t \rightarrow \infty$. Then, the tracking error of the flexible part can be written as

$$
\begin{aligned}
\ddot{\tilde{q}}_{f 2}= & -\left(\mathscr{M}_{d 41}-\mathscr{M}_{41}\right) \ddot{q}_{r d 1}-\left(\mathscr{H}_{d 4}-\mathscr{H}_{4}\right) \\
& -\mathscr{K}_{f 2} \widetilde{q}_{f 2}-\mathscr{D}_{f 2} \dot{\tilde{q}}_{f 2} .
\end{aligned}
$$

In state space form, the tracking error of the flexible part takes the same form as the one obtained in [20] and is given by the following expression:

$$
\dot{\tilde{x}}_{1}=\tilde{x}_{2}, \quad \dot{\tilde{x}}_{2}=\ddot{q}_{f d 2}-\ddot{q}_{f 2} .
$$

Let $\widetilde{x}_{f}=\left[\begin{array}{ll}\widetilde{x}_{1} & \widetilde{x}_{2}\end{array}\right]^{T}=\left[\begin{array}{ll}\widetilde{q}_{f 2} & \dot{\tilde{q}}_{f 2}\end{array}\right]^{T} ; \widetilde{q}_{f 2}=q_{f d 2}-q_{f 2}$. 


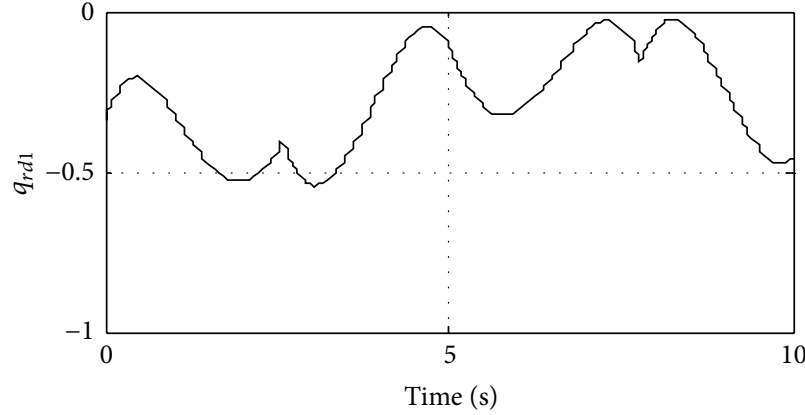

(a)

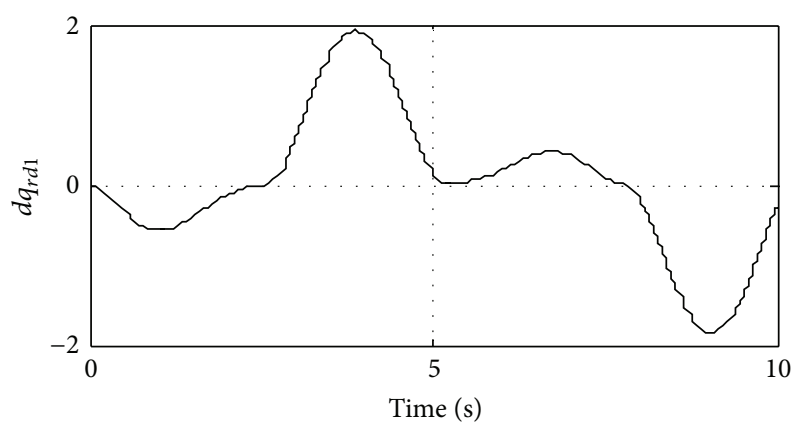

(c)

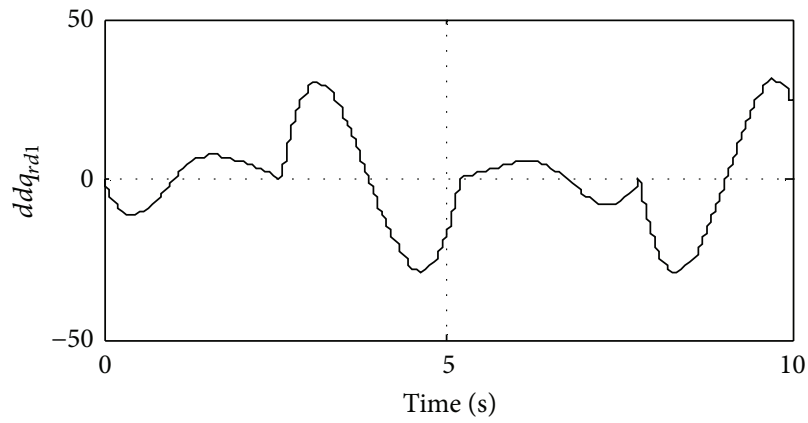

(e)

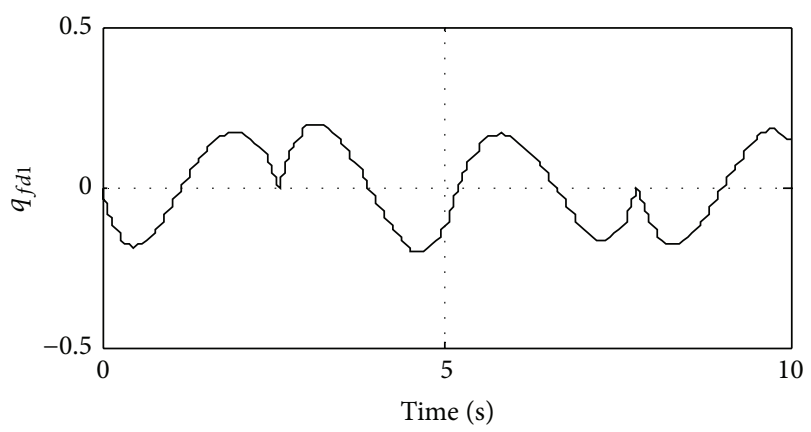

(g)

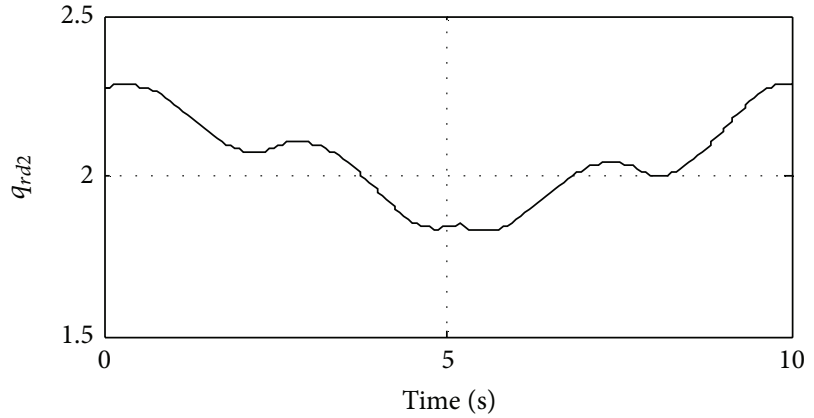

(b)

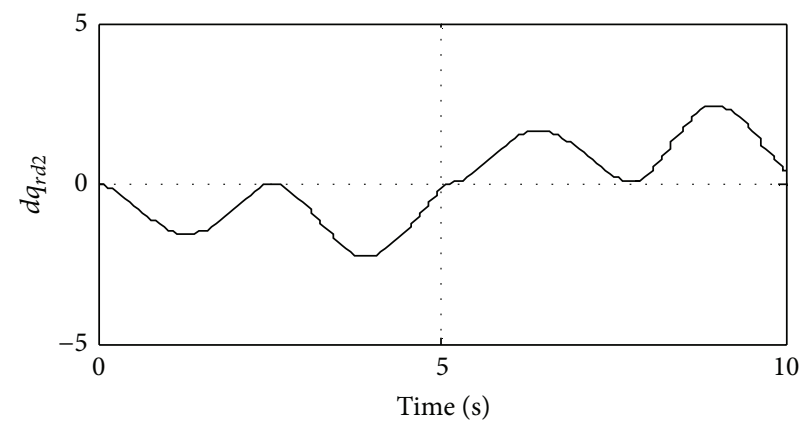

(d)

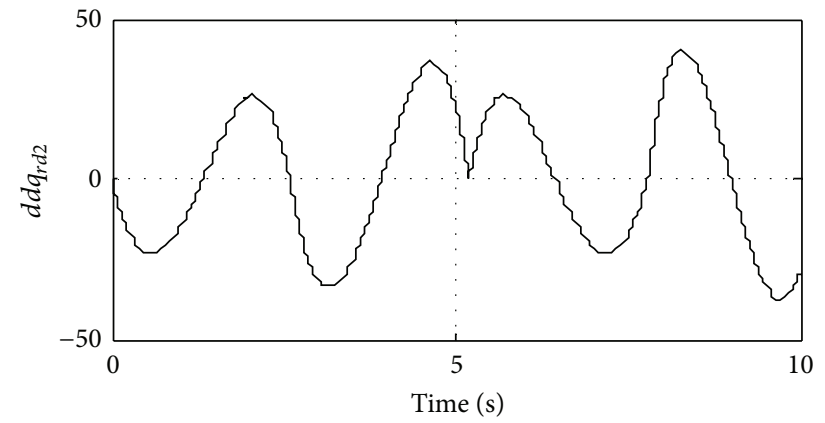

(f)

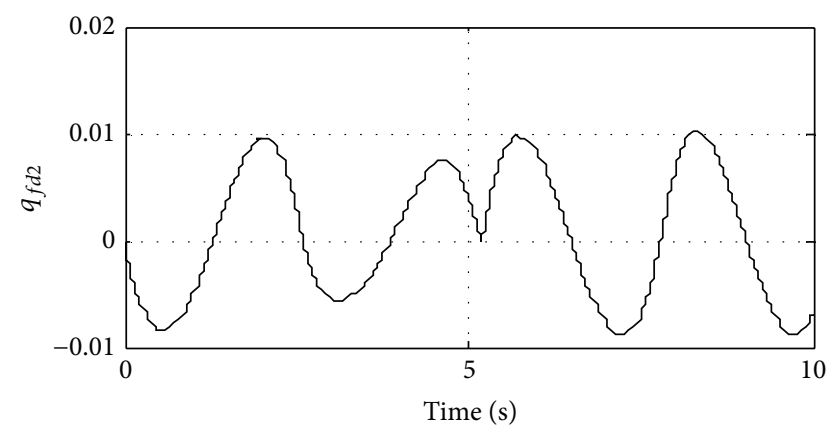

(h)

FIGURE 7: Desired joint space trajectories: (a)-(b) position trajectories of rigid coordinates, (c)-(d) velocity trajectories of rigid coordinates, (e)-(f) acceleration trajectories of rigid coordinates, and (g)-(h) position trajectories of flexible coordinates.

The critical value of $\alpha_{2}$ is selected such that $A_{f 2}$ is Hurwitz and the Nyquist diagram of $A_{f 2}$ is in the right half plan [20], where $A_{f 2}=\left[\begin{array}{cc}0 & 1 \\ -\mathscr{K}_{f 2} & -\mathscr{D}_{f 2}\end{array}\right] . \mathscr{K}_{f 2}$ and $\mathscr{D}_{f 2}$ are given in (62).

The same procedure is now applied, proceeding backward, to the first joint-link subsystem. Using (1) and (3), the equation of motion of the flexible part of the first link is given by

$$
\begin{gathered}
M_{31} \ddot{q}_{r 1}+M_{32} \ddot{q}_{r 2}+M_{33} \ddot{q}_{f 1}+M_{34} \ddot{q}_{f 2} \\
+H_{3}+D_{f 1} \dot{q}_{f 1}+K_{f 1} q_{f 1}=0 .
\end{gathered}
$$




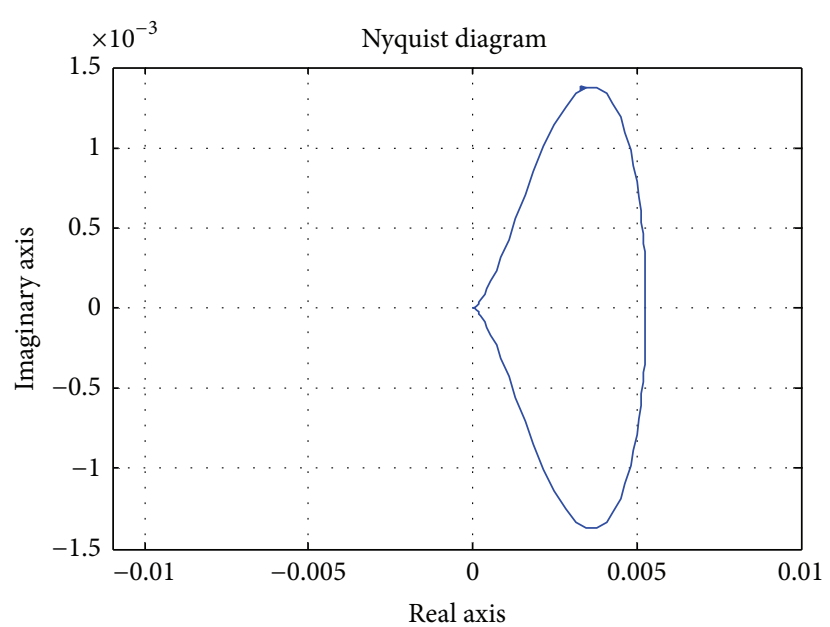

(a)

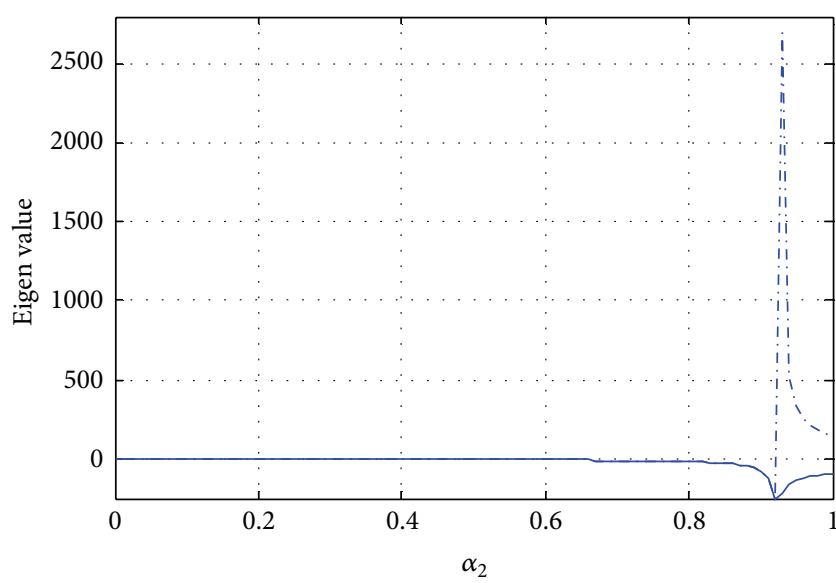

— First eigen value

...- Second eigen value

(b)

FIGURE 8: Stability of second link. (a) Nyquist diagram and (b) eigenvalues evolution [20].

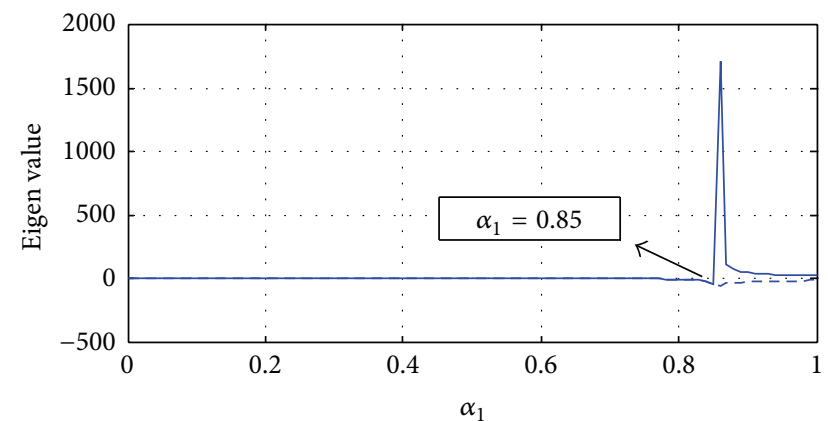

- First eigen value

- Second eigen value

FIGURE 9: Stability of first link: eigenvalues versus $\alpha_{1}$ [20].

Using the new generalized coordinate (38) and the new noncollocated output (40), the equation of motion becomes

$$
\begin{aligned}
M_{31} \ddot{y}_{1} & +M_{32} \ddot{q}_{r d 2}+\left(M_{33}-\alpha_{1} \beta_{1} M_{31}\right) \ddot{q}_{f 1} \\
& +M_{34} \ddot{q}_{f d 2}+H_{3}+D_{f 1} \dot{q}_{f 1}+K_{f 1} q_{f 1}=0 .
\end{aligned}
$$

Subtracting $\ddot{q}_{f 1}$ from (67), the internal dynamics are given as follows:

$$
\begin{aligned}
\ddot{q}_{f 1}= & -\mathscr{M}_{31} \ddot{y}_{1}-\mathscr{M}_{32} \ddot{q}_{r d 2}-\mathscr{M}_{34} \ddot{q}_{f d 2} \\
& -\mathscr{H}_{3}-\mathscr{K}_{f 1} q_{f 1}-\mathscr{D}_{f 1} \dot{q}_{f 1},
\end{aligned}
$$

where $\mathscr{M}_{31}=\left(M_{33}-\alpha_{1} \beta_{1} M_{31}\right)^{-1} M_{31} ; \mathscr{M}_{32}=\left(M_{33}-\right.$ $\left.\alpha_{1} \beta_{1} M_{31}\right)^{-1} M_{32} ; \mathscr{M}_{34}=\left(M_{33}-\alpha_{1} \beta_{1} M_{31}\right)^{-1} M_{34} ; \mathscr{H}_{3}=$ $\left(M_{33}-\alpha_{1} \beta_{1} M_{31}\right)^{-1} H_{3} ; \mathscr{K}_{f 1}=\left(M_{33}-\alpha_{1} \beta_{1} M_{31}\right)^{-1} K_{f 1} ;$ $\mathscr{D}_{f 1}=\left(M_{33}-\alpha_{1} \beta_{1} M_{31}\right)^{-1} D_{f 1}$, and $\alpha_{1}$ is chosen such as $\alpha_{1} \neq M_{33} / \beta_{1} M_{31}$.

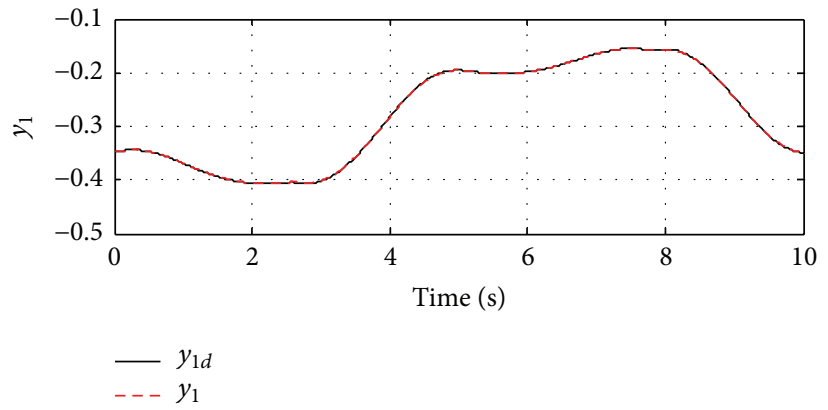

(a)

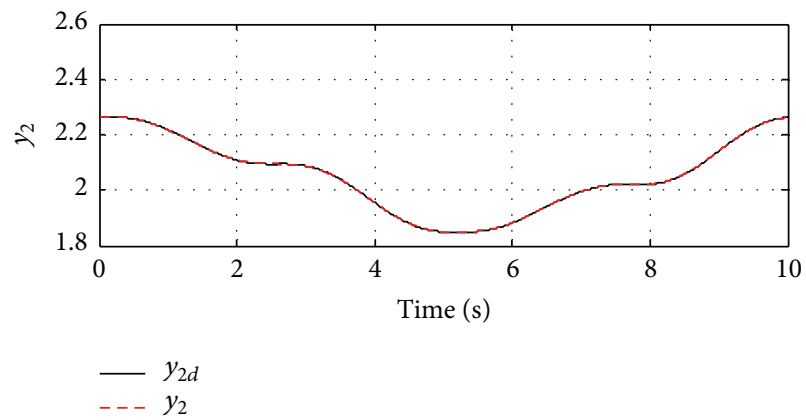

(b)

FIGURE 10: Tracking trajectories of noncollocated outputs.

In the state space form, the tracking error of the flexible part of the first link can be written as follows:

$$
\dot{\tilde{x}}_{f 1}=\dot{\tilde{q}}_{f 1}=\tilde{x}_{f 2}, \quad \dot{\tilde{x}}_{f 2}=\ddot{\tilde{q}}_{f 1}=\ddot{q}_{f d 1}-\ddot{q}_{f 1} .
$$

Let $\widetilde{x}_{f}=\left[\begin{array}{ll}\widetilde{x}_{f 1} & \widetilde{x}_{f 2}\end{array}\right]^{T}=\left[\begin{array}{ll}\widetilde{q}_{f 1} & \dot{\tilde{q}}_{f 1}\end{array}\right]^{T}$.

As already shown in the stability analysis of the rigid part, $\tilde{y}_{1}, \dot{\tilde{y}}_{1}$, and $\ddot{\tilde{y}}_{1} \rightarrow 0$ as $t \rightarrow \infty$. The error dynamics of 


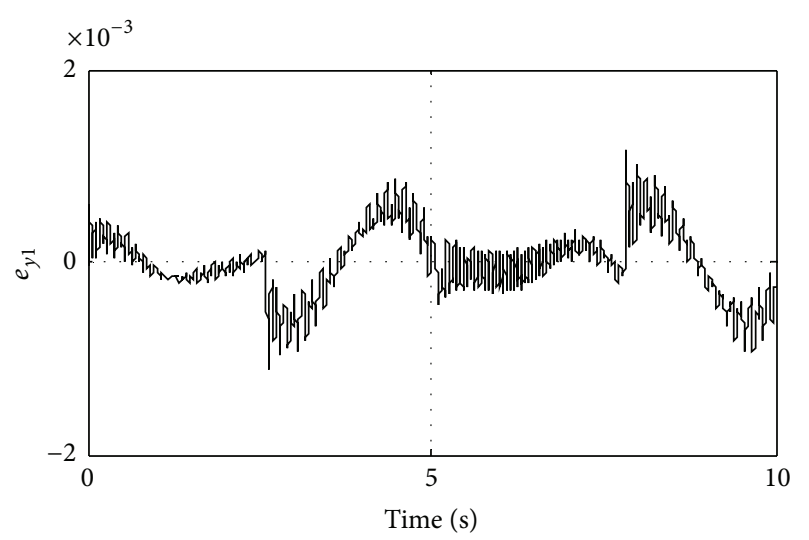

(a)

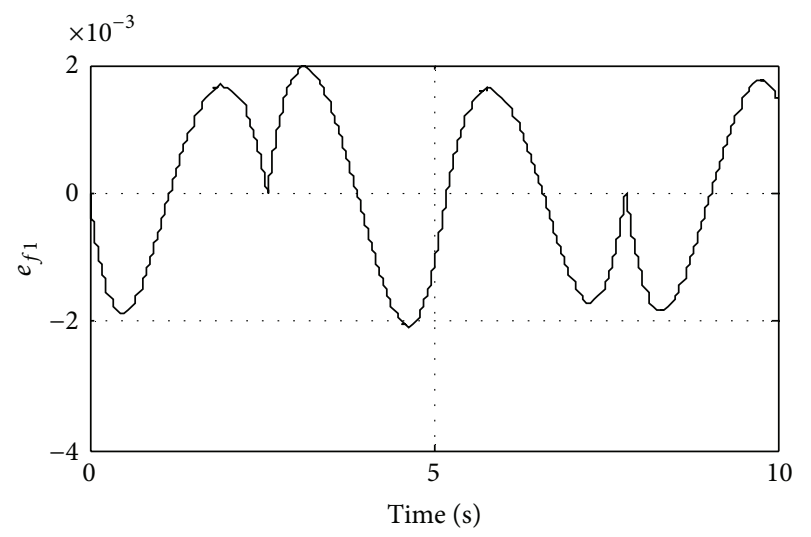

(c)

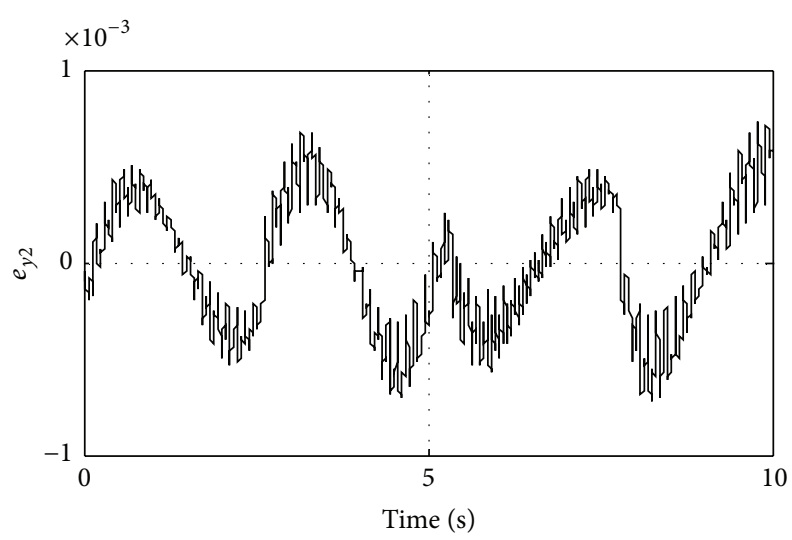

(b)

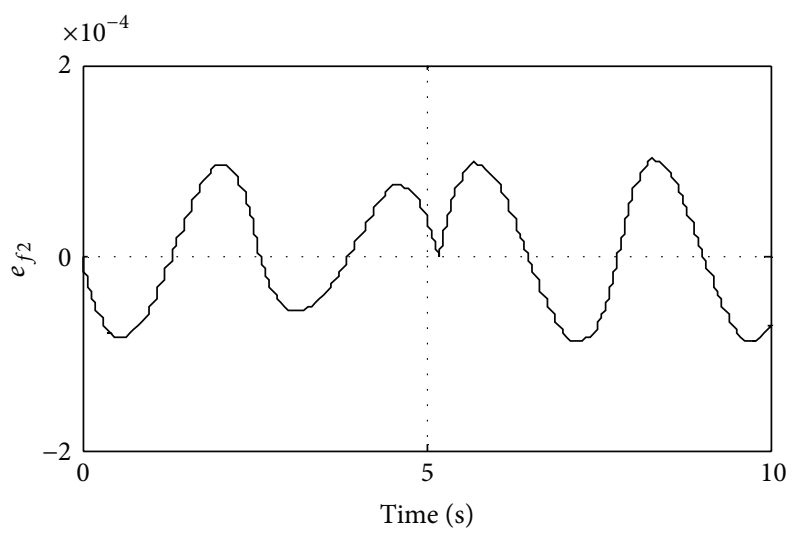

(d)

FIGURE 11: Tracking errors in joint space. (a)-(b) tracking errors of noncollocated outputs and (c)-(d) tracking errors of the flexible coordinates.

the flexible part (69) take the same form as the one obtained in [20].

The critical value of $\alpha_{1}$ is selected when the eigenvalues of $A_{f 1}$ change their signs from negative to positive; for example, the internal dynamics become instable [20], where $A_{f 1}=$ $\left[-\frac{0}{-K_{f 1}}-\frac{1}{D_{f 1}}\right]$.

\section{Simulation Results}

The two-flexible-link manipulator shown in Figure 2 is used to test the control strategy performance. Using the trial and error method, the controllers' gains are chosen as $K_{p 1}=$ $12, K_{p 2}=10, K_{d 1}=15$, and $K_{d 2}=18$.

In Sections 3 and 4 , the theoretical development of the control law and stability analysis are given in the general case, that is, for any higher order. In the simulation case, a finite order of Taylor series is fixed. In this section, for simplicity, the Taylor series is limited to the first order. Then, $R_{M_{n j}}=$ $R_{C_{n j}}=R_{G_{n}}=0$.

Table 1 presents the parameters of the two-flexible-link manipulator.

Simulation results are given Figures 4-14.

For two-flexible-link manipulators, the desired workspace trajectory is chosen as lozenge form. The results of the inverse kinematics problem are given in Figures 4-7. Workspace trajectory is shown in Figures 4 and 5, virtual space trajectories are given in Figure 6, and joint space trajectories are shown in Figure 7. According to simulation results, the workspace desired trajectory was well transformed to the joint space using the virtual space and the quasi-static approach. For the second subsystem, the Nyquist diagram and the evolution of eigenvalues of $A_{f_{2}}$ are given in Figure 8. For the first subsystem, the eigenvalues evolution of $A_{f 1}$ is given in Figure 9. Using the system parameters given in Table 1, the critical value of the second and first links are $\alpha_{2 c}=0.92$ and $\alpha_{1 c}=0.85$, respectively. The simulation results were obtained with $\alpha_{2}=0.9<\alpha_{2 c}$ and $\alpha_{1}=0.8<\alpha_{1 c}$. Note that these values satisfy the conditions given in (30) and (38).

A good tracking performance of the new noncollocated output was obtained, as shown in Figure 10. This tracking is confirmed by Figure 11, which shows the tracking error. In the workspace, a good tracking performance is shown in Figure 12, and the tracking error, shown in Figure 13, does not exceed $2 \mathrm{~mm}$ in $x$-position and $y$-position. The good tracking in the workspace is confirmed in Figure 14 that shows the tracking of the desired lozenge. Thus, we can conclude that the control strategy was effective to ensure the tracking of the noncollocated output and to reduce vibrations at 
TABLE 1: System parameters.

\begin{tabular}{lc}
\hline Parameter & Value \\
\hline Hub inertia $\left(J h_{i}\right)$ & $0.1 \mathrm{~kg} \cdot \mathrm{m}^{2}$ \\
Link length $\left(L_{i}\right)$ & $0.5 \mathrm{~m}$ \\
Link linear density $(\rho)$ & $1 \mathrm{~kg} / \mathrm{m}$ \\
Link rigidity $\left(E I_{i}\right)$ & $10 \mathrm{~N} \cdot \mathrm{m}^{2}$ \\
Link mass $\left(m_{i}\right)$ & $0.5 \mathrm{~kg}$ \\
Payload mass $\left(m_{p}\right)$ & $0.1 \mathrm{~kg}$ \\
Payload inertia $\left(J_{p}\right)$ & $0.0005 \mathrm{~kg} \cdot \mathrm{m}^{2}$ \\
\hline
\end{tabular}
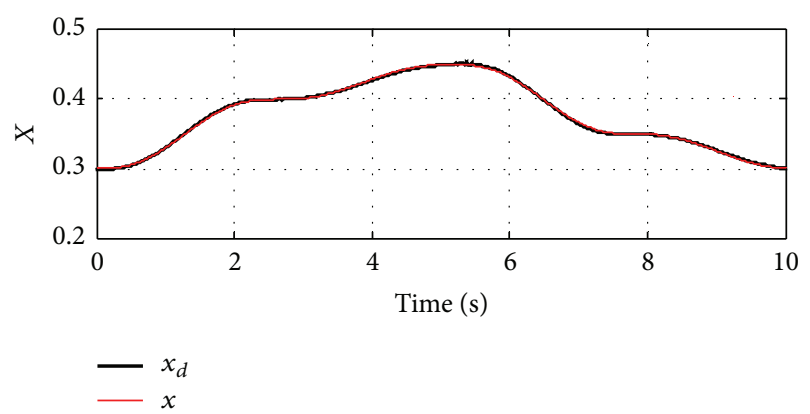

(a)

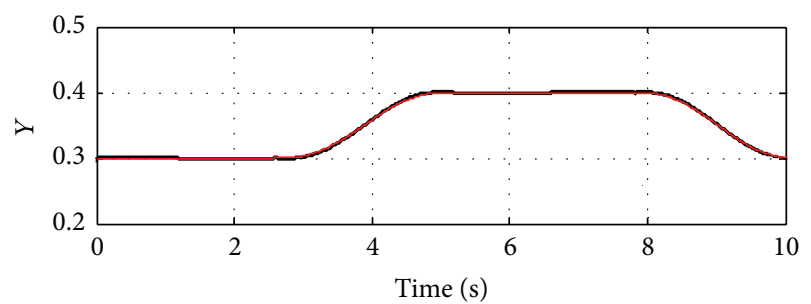

$-y_{d}$
$-y$

(b)

FIGURE 12: Tracking in the workspace. (a) $x$-position tracking and (b) $y$-position tracking.

the extremity; this explains the satisfactory results obtained using this approach.

\section{Conclusion and Future Work}

This paper presents a nonlinear distributed control for twoflexible-link manipulator. For the inverse dynamics, a virtual space, linked with the workspace by a simple kinematics relation as in rigid manipulators, and a quasi-static approach were used. Using this transformation procedure, a workspace desired trajectory (lozenge) has been successfully transformed to the joint space. The distributed control strategy presented in this paper uses the output redefinition technique and consists of stabilizing the flexible manipulators starting with the last joint and flexible link and going backward until the first joint and link. Lyapunov theory was used to prove the asymptotical stability. An adaptive version of this control strategy will be investigated in future work.

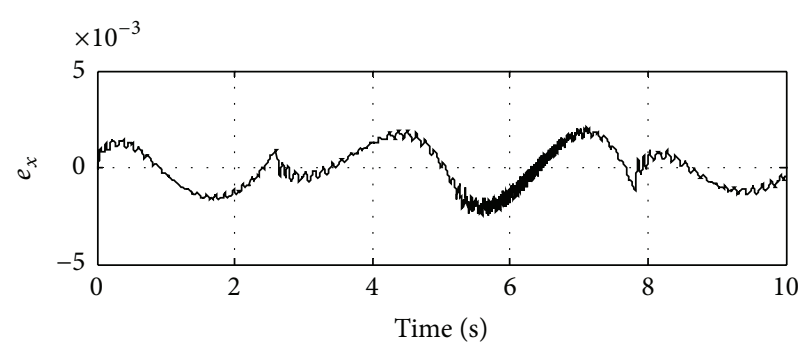

(a)

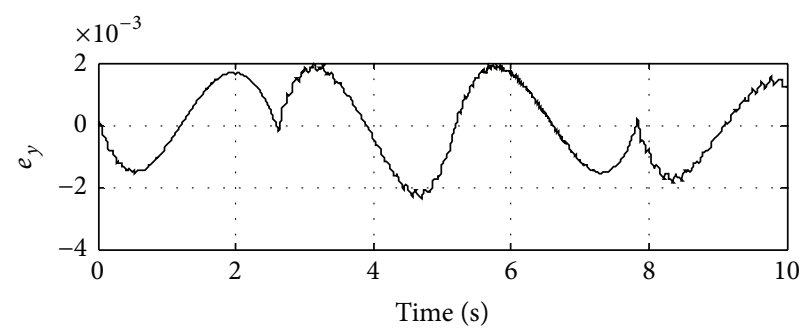

(b)

FIGURE 13: Workspace tracking errors. (a) $x$-position tracking errors and (b) $y$-position tracking errors.

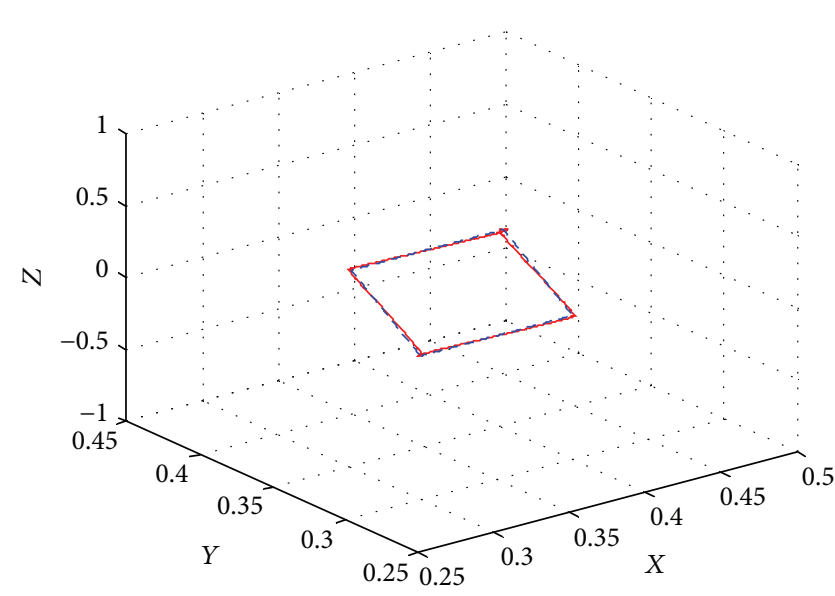

FIGURE 14: $X-Y$ workspace tracking.

\section{Appendices}

\section{A. Proof of Proposition 1}

Using (3) and (5), the dynamical model can be written as follows:

$$
\begin{aligned}
& {\left[\begin{array}{ll}
M_{r r} & M_{r f} \\
M_{r f}^{T} & M_{f f}
\end{array}\right]\left[\begin{array}{l}
\ddot{q}_{r} \\
\ddot{q}_{f}
\end{array}\right]+\left[\begin{array}{ll}
C_{r r} & C_{r f} \\
C_{r f}^{T} & C_{f f}
\end{array}\right]\left[\begin{array}{l}
\dot{q}_{r} \\
\dot{q}_{f}
\end{array}\right]} \\
& \quad+\left[\begin{array}{cc}
0 & 0 \\
0 & D_{f f}
\end{array}\right]\left[\begin{array}{l}
\dot{q}_{r} \\
\dot{q}_{f}
\end{array}\right]+\left[\begin{array}{cc}
0 & 0 \\
0 & K_{f f}
\end{array}\right]\left[\begin{array}{l}
q_{r} \\
q_{f}
\end{array}\right]=\left[\begin{array}{l}
I \\
0
\end{array}\right] \tau .
\end{aligned}
$$


Equation (A.1) can be written as

$$
\begin{gathered}
M_{r r} \ddot{q}_{r}+M_{r f} \ddot{q}_{f}+C_{r r} \dot{q}_{r}+C_{r f} \dot{q}_{f}=\tau, \\
M_{r f}^{T} \ddot{q}_{r}+M_{f f} \ddot{q}_{f}+C_{r f}^{T} \dot{q}_{r}+C_{f f} \dot{q}_{f}+D_{f f} \dot{q}_{f}+K_{f f} q_{f}=0 .
\end{gathered}
$$

Using the outputs redefinition (31) and (40), we can write

$$
q_{r}=y-\alpha \beta q_{f}
$$

where $q_{r}=\left[\begin{array}{ll}q_{r 1} & q_{r 2}\end{array}\right]^{T} ; q_{f}=\left[\begin{array}{ll}q_{f 1} & q_{f 2}\end{array}\right]^{T} ; y=\left[\begin{array}{ll}y_{1} & y_{2}\end{array}\right]^{T}$ and $\alpha \beta=\left[\begin{array}{cc}\alpha_{1} \beta_{1} & 0 \\ 0 & \alpha_{2} \beta_{2}\end{array}\right]$. Then, the dynamical model becomes

$$
\begin{gathered}
M_{r r} \ddot{y}+\mathscr{M}_{r f} \ddot{q}_{f}+C_{r r} \dot{y}+\mathscr{C}_{r f} \dot{q}_{f}=\tau \\
M_{r f}^{T} \ddot{y}+\mathscr{M}_{f f} \ddot{q}_{f}+C_{r f}^{T} \dot{y}+\mathscr{C}_{f f} \dot{q}_{f}+D_{f f} \dot{q}_{f}+K_{f f} q_{f}=0,
\end{gathered}
$$

where $\mathscr{M}_{r f}=M_{r f}-\alpha \beta M_{r r} ; \mathscr{C}_{r f}=C_{r f}-\alpha \beta C_{r r} ; \mathscr{M}_{f f}=$ $M_{f f}-\alpha \beta M_{r f}^{T} ; \mathscr{C}_{f f}=C_{f f}-\alpha \beta C_{r f}^{T}$.

Deducing $\ddot{q}_{f}$ from (A.5), we get the following internal dynamics:

$$
\ddot{q}_{f}=-\mathscr{M}_{f f}^{-1}\left(M_{r f}^{T} \ddot{y}+C_{r f}^{T} \dot{y}+\mathscr{C}_{f f} \dot{q}_{f}+D_{f f} \dot{q}_{f}+K_{f f} q_{f}\right) .
$$

Inserting (A.6) in (A.4), we obtain

$$
M_{r}^{*} \ddot{y}+C_{r}^{*} \dot{y}+C_{r f}^{*} \dot{q}_{f}+D_{f}^{*} \dot{q}_{f}+K_{f}^{*} q_{f}=\tau,
$$

where $M_{r}^{*}=M_{r r}-\mathscr{M}_{r f} \mathscr{M}_{f f}^{-1} M_{r f}^{T} ; C_{r}^{*}=C_{r r}-\mathscr{M}_{r f} \mathscr{M}_{f f}^{-1} C_{r f}^{T}$; $C_{r f}^{*}=\mathscr{C}_{r f}-\mathscr{M}_{r f} \mathscr{M}_{f f}^{-1} \mathscr{C}_{f f} ; D_{f}^{*}=-\mathscr{M}_{r f} \mathscr{M}_{f f}^{-1} D_{f f}$ and $K_{f}^{*}=$ $-\mathscr{M}_{r f} \mathscr{M}_{f f}^{-1} K_{f f}$.

The models using the collocated output (3) and the new output (A.4)-(A.5) are linked by a nonsingular positive transformation matrix as follows:

$$
q_{\alpha}=\left[\begin{array}{c}
q_{r}+\alpha \beta q_{f} \\
q_{f}
\end{array}\right]=T q,
$$

where $q_{\alpha}=\left[\begin{array}{ll}y & q_{f}\end{array}\right]^{T}$ and $T=\left[\begin{array}{cc}1 & \alpha \beta \\ 0 & 1\end{array}\right]$.

Using the transformation $T$, the mass matrix becomes

$$
\begin{aligned}
M(q) & =\left[\begin{array}{ll}
M_{r r}(q) & M_{r f}(q) \\
M_{r f}^{T}(q) & M_{f f}(q)
\end{array}\right] \stackrel{q_{\alpha}=T q}{\longrightarrow} \mathscr{M}\left(q_{\alpha}\right) \\
& =\left[\begin{array}{ll}
M_{r r}\left(q_{\alpha}\right) & M_{r f}\left(q_{\alpha}\right) \\
M_{r f}^{T}\left(q_{\alpha}\right) & \mathscr{M}_{f f}\left(q_{\alpha}\right)
\end{array}\right],
\end{aligned}
$$

where $\mathscr{M}_{r f}\left(q_{\alpha}\right)=M_{r f}\left(q_{\alpha}\right)-\alpha \beta M_{r r}\left(q_{\alpha}\right)$ and $\mathscr{M}_{f f}\left(q_{\alpha}\right)=$ $M_{f f}\left(q_{\alpha}\right)-\alpha \beta M_{r f}^{T}\left(q_{\alpha}\right)$ or in a matrix form:

$$
\mathscr{M}\left(q_{\alpha}\right)=M\left(T^{-1} q_{\alpha}\right) T^{-1} .
$$

The parameter $\alpha$ is selected such that $\mathscr{M}$ is positive definite matrix. Then, using (P1) and (P2), $\mathscr{M}_{r r}, \mathscr{M}_{f f}$, and $\mathscr{M}_{i i}$ are positive definite matrices. The same transformation matrix can be used for the Coriolis matrix as follows:

$$
\mathscr{C}\left(q_{\alpha}, \dot{q}_{\alpha}\right)=C\left(T^{-1} q_{\alpha}, T^{-1} \dot{q}_{\alpha}\right) T^{-1}
$$

where $\mathscr{C}\left(q_{\alpha}\right)=\left[\begin{array}{cc}C_{r r} & \bar{C}_{r f} \\ C_{r f}^{T} & \bar{C}_{f f}\end{array}\right] ; \mathscr{C}_{r f}=C_{r f}-\alpha \beta C_{r r}$ and $\mathscr{C}_{f f}=$ $C_{f f}-\alpha \beta C_{r f}^{T}$.

From (P4), by multiplying (6) by a constant matrix $T^{-1}$, we can write

$$
X^{T}\left(\dot{M}\left(q_{\alpha}\right)-2 \mathscr{C}\left(q_{\alpha}, \dot{q}_{\alpha}\right)\right) X=0, \quad \forall X \in \mathscr{R}^{n}
$$

The Schur complement of the positive definite matrix $\mathscr{M}_{f f}$ given by

$$
M_{r}^{*}=M_{r r}-\mathscr{M}_{r f} \mathscr{M}_{f f}^{-1} M_{r f}^{T}
$$

is positive [22]. Then, from (P2), the diagonal elements of $M_{r}^{*}$ are also positive.

The Schur complement of $\mathscr{C}_{f f}$ is given by the following equation:

$$
C_{r}^{*}=C_{r r}-\mathscr{C}_{r f} \mathscr{C}_{f f}^{-1} C_{r f}^{T}
$$

Using the dynamical model of the two-flexible-link manipulator [20], we can write

$$
X^{T}\left(\dot{M}_{r}^{*}-2 C_{r}^{*}\right) X=0, \quad \forall X \in \mathscr{R}^{n}
$$

\section{B. Proof of Proposition 2}

In the following, we study the error dynamics given by (55). In the first step, we investigate the following elements of the error dynamics: $\left[M_{r d}^{*}(Q) \ddot{y}^{*}-M_{r d}^{*}\left(q_{\alpha}\right) \ddot{y}\right],\left[M_{r n d}^{*}(Q) \ddot{y}_{d}-\right.$ $\left.M_{r n d}^{*}\left(q_{\alpha}\right) \ddot{y}\right],\left[C_{r}^{*}(Q, \dot{Q}) \dot{y}_{d}-C_{r}^{*}\left(q_{\alpha}, \dot{q}_{\alpha}\right) \dot{y}\right]$, and $\left[C_{r f}^{*}(Q, \dot{Q}) \dot{q}_{f}-\right.$ $\left.C_{r f}^{*}\left(q_{\alpha}, \dot{q}_{\alpha}\right) \dot{q}_{f}\right]$.

The first element is developed as

$$
\begin{aligned}
& {\left[M_{r d}^{*}(Q) \ddot{y}^{*}-M_{r d}^{*}\left(q_{\alpha}\right) \ddot{y}\right] } \\
&=\left[\begin{array}{cc}
M_{11}^{*}\left(Q_{1}\right) & 0 \\
0 & M_{22}^{*}\left(Q_{2}\right)
\end{array}\right]\left[\begin{array}{l}
\ddot{y}_{1}^{*} \\
\ddot{y}_{2}^{*}
\end{array}\right] \\
&-\left[\begin{array}{cc}
M_{11}^{*}\left(q_{\alpha}\right) & 0 \\
0 & M_{22}^{*}\left(q_{\alpha}\right)
\end{array}\right]\left[\begin{array}{l}
\ddot{y}_{1} \\
\ddot{y}_{2}
\end{array}\right] \\
&= {\left[\begin{array}{c}
M_{11}^{*}\left(Q_{1}\right) \ddot{y}_{1}^{*}-M_{11}^{*}\left(q_{\alpha}\right) \ddot{y}_{1} \\
M_{22}^{*}\left(Q_{2}\right) \ddot{y}_{2}^{*}-M_{22}^{*}\left(q_{\alpha}\right) \ddot{y}_{2}
\end{array}\right], }
\end{aligned}
$$

where $q_{\alpha}=\left[\begin{array}{llll}y_{1} & y_{2} & q_{f 1} & q_{f 2}\end{array}\right]^{T}, Q_{1}=\left[\begin{array}{llll}y_{1} & q_{r d 2} & q_{f 1} & q_{f d 2}\end{array}\right]^{T}$, $Q_{2}=\left[\begin{array}{llll}q_{r d 1} & y_{2} & q_{f d 1} & q_{f 2}\end{array}\right]^{T}$, and $q_{2}=\left[\begin{array}{ll}q_{r 2} & q_{f 2}\end{array}\right]^{T}$. 
Using Taylor series, we can write

$$
\begin{aligned}
M_{11}^{*}\left(q_{\alpha}\right)= & M_{11}^{*}\left(Q_{1}\right)+\left.\frac{\partial M_{11}\left(q_{\alpha}\right)}{\partial q_{2}}\right|_{q_{d 2}}\left(q_{2}-q_{d 2}\right)+R_{M_{11}}\left(\widetilde{q}_{2}\right) \\
= & M_{11}^{*}\left(Q_{1}\right)-\left.\frac{\partial M_{11}\left(q_{\alpha}\right)}{\partial q_{r 2}}\right|_{q_{r d 2}} \widetilde{q}_{r 2}-\left.\frac{\partial M_{11}\left(q_{\alpha}\right)}{\partial q_{f 2}}\right|_{q_{f d 2}} \\
& \times \tilde{q}_{f 2}+R_{M_{11}}\left(\widetilde{q}_{2}\right) \\
= & M_{11}^{*}\left(Q_{1}\right)-\delta M_{11},
\end{aligned}
$$

where

$$
\begin{aligned}
\delta M_{11}= & \left.\frac{\partial M_{11}\left(q_{\alpha}\right)}{\partial q_{r 2}}\right|_{q_{r d 2}} \tilde{q}_{r 2}+\left.\frac{\partial M_{11}\left(q_{\alpha}\right)}{\partial q_{f 2}}\right|_{q_{f d 2}} \\
& \times \delta \widetilde{q}_{f 2}-R_{M_{11}}\left(\widetilde{q}_{2}\right) .
\end{aligned}
$$

$R_{M_{11}}$ are the high order terms of the Taylor series for $M_{11}^{*}\left(q_{\alpha}\right)$. Then,

$$
M_{11}^{*}\left(Q_{1}\right)=M_{11}^{*}\left(q_{\alpha}\right)+\delta M_{11}^{*} .
$$

The Taylor series is also applied to the following elements to obtain

$$
\begin{gathered}
M_{r d}^{*}(Q)=M_{r d}^{*}\left(q_{\alpha}\right)+\delta M_{r d}^{*}, \\
M_{r n d}^{*}(Q)=M_{r n d}^{*}\left(q_{\alpha}\right)+\delta M_{r n d}^{*}, \\
C_{r}^{*}(Q, \dot{Q})=C_{r}^{*}\left(q_{\alpha}, \dot{q}_{\alpha}\right)+\delta C_{r}^{*}, \\
C_{r f}^{*}(Q, \dot{Q})=C_{r f}^{*}\left(q_{\alpha}, \dot{q}_{\alpha}\right)+\delta C_{r f}^{*} .
\end{gathered}
$$

Then, the previous elements in the error dynamics become

$$
\begin{gathered}
M_{r d}^{*}(Q) \ddot{y}^{*}-M_{r d}^{*}\left(q_{\alpha}\right) \ddot{y} \\
=M_{r d}^{*}\left(q_{\alpha}\right)\left[\ddot{y}^{*}-\ddot{y}\right]+\delta M_{r d}^{*} \ddot{y}^{*} \\
=M_{r d}^{*}\left(q_{\alpha}\right) \ddot{\tilde{y}}^{*} \lambda M_{r d}^{*}\left(q_{\alpha}\right) \dot{\tilde{y}}+\delta M_{r d}^{*} \ddot{y}^{*}, \\
{\left[M_{r n d}^{*}(Q) \ddot{y}_{d}-M_{r n d}^{*}\left(q_{\alpha}\right) \ddot{y}\right]=M_{r n d}^{*}\left(q_{\alpha}\right) \ddot{\tilde{y}}+\delta M_{r n d}^{*} \ddot{y}_{d},} \\
{\left[C_{r}^{*}(Q, \dot{Q}) \dot{y}_{d}-C_{r}^{*}\left(q_{\alpha}, \dot{q}_{\alpha}\right) \dot{y}\right]=C_{r}^{*}\left(q_{\alpha}, \dot{q}_{\alpha}\right) \dot{\tilde{y}}+\delta C_{r}^{*} \dot{y}_{d} .}
\end{gathered}
$$

Inserting these expressions into the error dynamics (55), they become

$$
\begin{aligned}
K_{p} \tilde{y} & +K_{d} \dot{\tilde{y}}+M_{r d}^{*}\left(q_{\alpha}\right) \ddot{\tilde{y}}+\lambda M_{r d}^{*}\left(q_{\alpha}\right) \dot{\tilde{y}} \\
& +M_{r n d}^{*}\left(q_{\alpha}\right) \ddot{\tilde{y}}+C_{r}^{*}\left(q_{\alpha}, \dot{q}_{\alpha}\right) \dot{\tilde{y}}+\delta M_{r d}^{*} \ddot{y}^{*} \\
& +\delta M_{r n d}^{*} \ddot{y}_{d}+\delta C_{r}^{*} \dot{y}_{d}+\delta C_{r f}^{*}+\delta \tau=0 .
\end{aligned}
$$

Using the expression of $\delta \tau$ given in (48), the error dynamics become

$$
K_{p} \tilde{y}+K_{d} \dot{\tilde{y}}+M_{r}^{*}\left(q_{\alpha}\right) \ddot{\tilde{y}}+C_{r}^{*}\left(q_{\alpha}, \dot{q}_{\alpha}\right) \dot{\tilde{y}}+\lambda M_{r d}^{*}\left(q_{\alpha}\right) \dot{\tilde{y}}=0 .
$$

\section{References}

[1] G. Hirzinger, N. Sporer, M. Schedl, J. Butterfaß, and M. Grebenstein, "Torque-controlled lightweight arms and articulated hands: do we reach technological limits now?" International Journal of Robotics Research, vol. 23, no. 4-5, pp. 331-340, 2004.

[2] U. Hagn, M. Nickl, S. Jörg et al., "The DLR MIRO: a versatile lightweight robot for surgical applications," Industrial Robot, vol. 35, no. 4, pp. 324-336, 2008.

[3] R. H. Cannon Jr. and E. Schmitz, "Initial experiments on the end-point control of a flexible one-link robot," International Journal of Robotics Research, vol. 3, no. 3, pp. 62-75, 1984.

[4] A. De Luca and B. Siciliano, "Trajectory control of a non-linear one-link flexible arm," International Journal of Control, vol. 50, no. 5, pp. 1699-1715, 1989.

[5] P. Bigras, M. Saad, and J. O'Shea, "Convergence analysis of an inverse flexible manipulator model algorithm," Journal of Vibration and Control, vol. 9, no. 10, pp. 1141-1158, 2003.

[6] L. Cuvillon, "A mutivariable methodology for fast visual servoing of flexible manipulators moving in a restricted workspace," Advanced Robotics, vol. 26, pp. 1771-1797, 2012.

[7] M. Mosayebi, "A nonlinear high gain observer based inputoutput control of flexible link manipulator," Mechanics Research Communications, vol. 45, pp. 34-41, 2012.

[8] C. C. De Wit, in Theory of Robot Control, Springer, New York, NY, USA, 1996.

[9] B. Siciliano and O. Khatib, in Handbook of Robotics, p. 1611, Springer, 2008.

[10] M. Moallem, R. V. Patel, and K. Khorasani, "Nonlinear tipposition tracking control of a flexible-link manipulator: theory and experiments," Automatica, vol. 37, no. 11, pp. 1825-1834, 2001.

[11] P. Bigras, M. Saad, and J. O'Shea, "Robust trajectory control in the workspace of a class of flexible robots," Journal of Robotic Systems, vol. 18, no. 6, pp. 275-288, 2001.

[12] B. Siciliano and W. J. Book, " singular perturbation approach to control of lightweight flexible manipulators," International Journal of Robotics Research, vol. 7, no. 4, pp. 79-90, 1988.

[13] Y. Zhang, T. Yang, and Z. Sun, "Neuro-sliding-mode control of flexible-link manipulators based on singularly perturbed model," Tsinghua Science and Technology, vol. 14, no. 4, pp. 444451, 2009.

[14] A. De Luca and B. Sicilicano, "Inversion-based nonlinear control of robot arms with flexible links," Journal of Guidance, Control, and Dynamics, vol. 16, no. 6, pp. 1169-1176, 1993.

[15] K. S. Fu, R. C. Gonzalez, and C. S. G. Lee, in Robotics: Control, Sensing, Vision, and Intelligence, McGraw-Hill, New York, NY, USA, 1987.

[16] F. Khorrami, I. Zeinoun, and E. Tome, "Experimental results on active control of flexible-link manipulators with embedded piezoceramics," in Proceedings of the IEEE International Conference on Robotics and Automation, pp. 222-227, May 1993.

[17] D. Sun and J. K. Mills, "Control of a rotating cantilever beam using a torque actuator and a distributed piezoelectric polymer actuator," Applied Acoustics, vol. 63, no. 8, pp. 885-899, 2002.

[18] R. Fareh, M. R. Saad, and M. Saad, "Workspace distributed realtime control of rigid manipulators," Journal of Vibration and Control, 2012.

[19] R. Fareh, M. R. Saad, and M. Saad, "Workspace tracking trajectory for 7-DOF ANAT robot using a hierarchical control strategy," in Proceedings of the 20th Mediterranean Conference 
on Control and Automation, pp. 122-127, Barcelona, Spain, 2012 July.

[20] R. Fareh, M. R. Saad, and M. Saad, "Workspace trajectory tracking control for two-flexible-link manipulator through output redefinition," International Journal of Modelling, Identification and Control, vol. 18, pp. 119-135, 2013.

[21] A. De Luca and B. Siciliano, "Closed-form dynamic model of planar multilink lightweight robots," IEEE Transactions on Systems, Man and Cybernetics, vol. 21, no. 4, pp. 826-839, 1991.

[22] R. Bhatia, in Positive Definite Matrices, Princeton University Press, Princeton, NJ, USA, 2007.

[23] A. De Luca and B. Siciliano, "Explicit dynamic modeling of a planar two-link flexible manipulator," in Proceedings of the 29th IEEE Conference on Decision and Control (Cat. No.90CH29173), pp. 528-530, New York, NY, USA, December 1990.

[24] M. W. Spong and M. Vidyasagar, in Robot Dynamics and Control, Wiley, New York, NY, USA, 1989. 

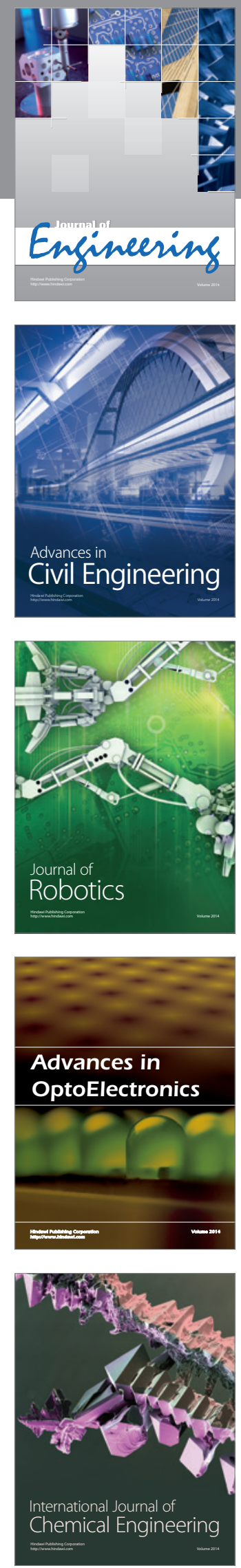

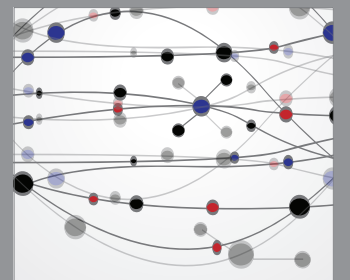

The Scientific World Journal
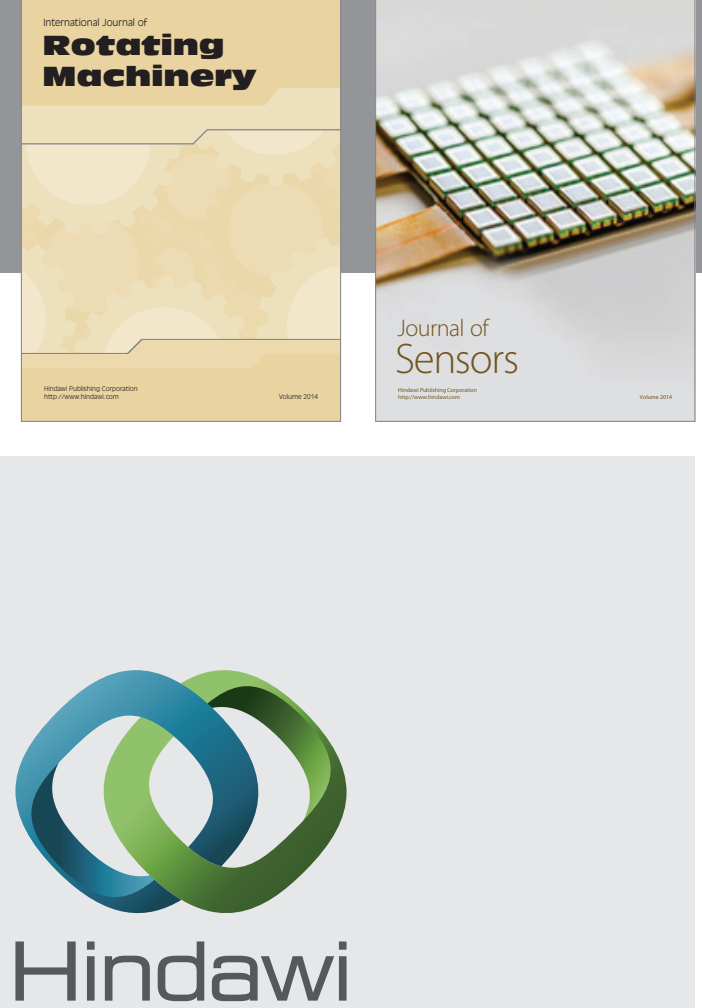

Submit your manuscripts at http://www.hindawi.com
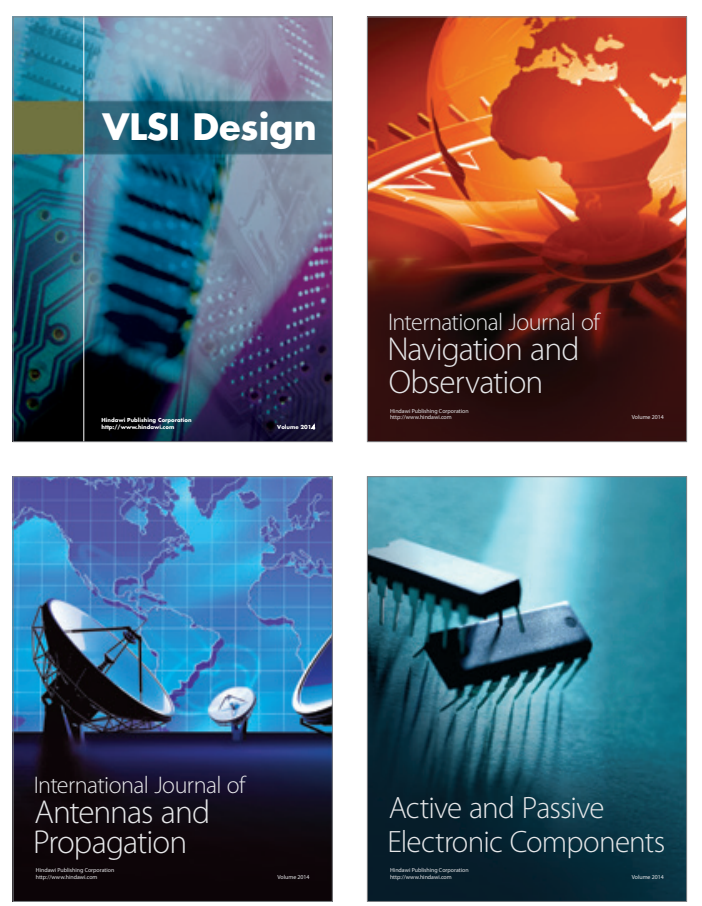
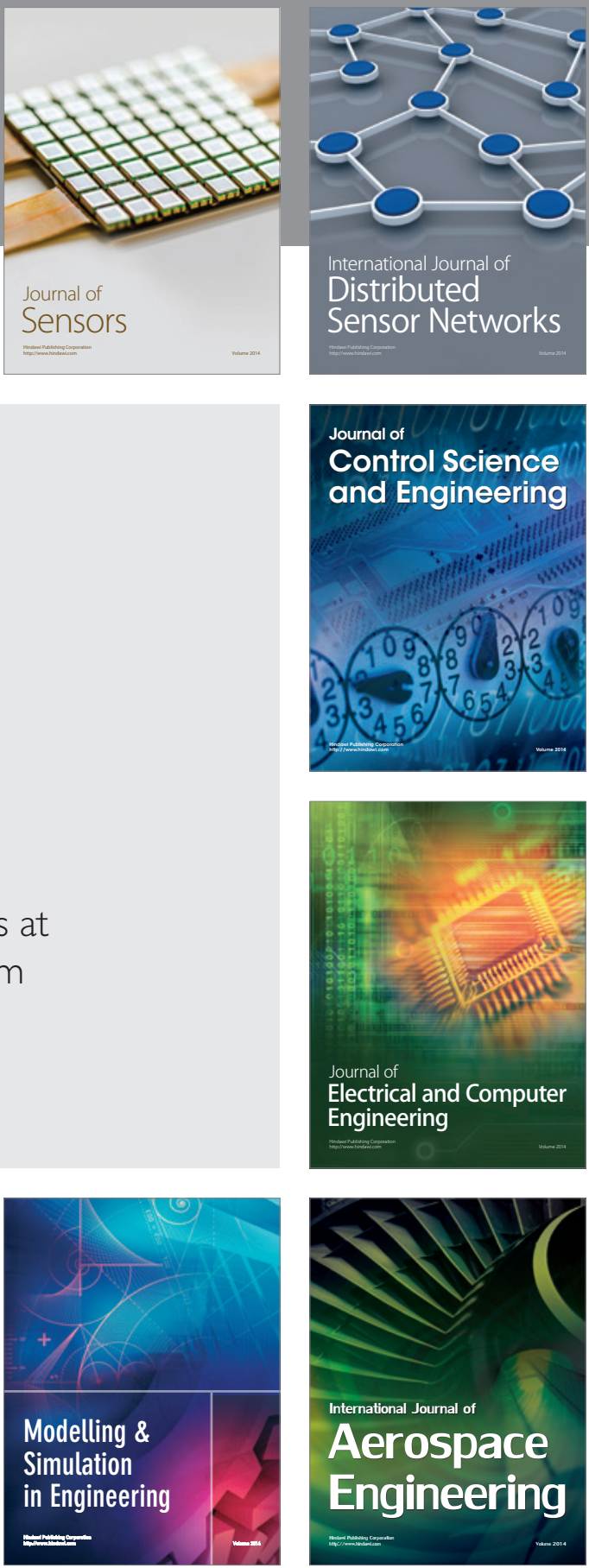

Journal of

Control Science

and Engineering
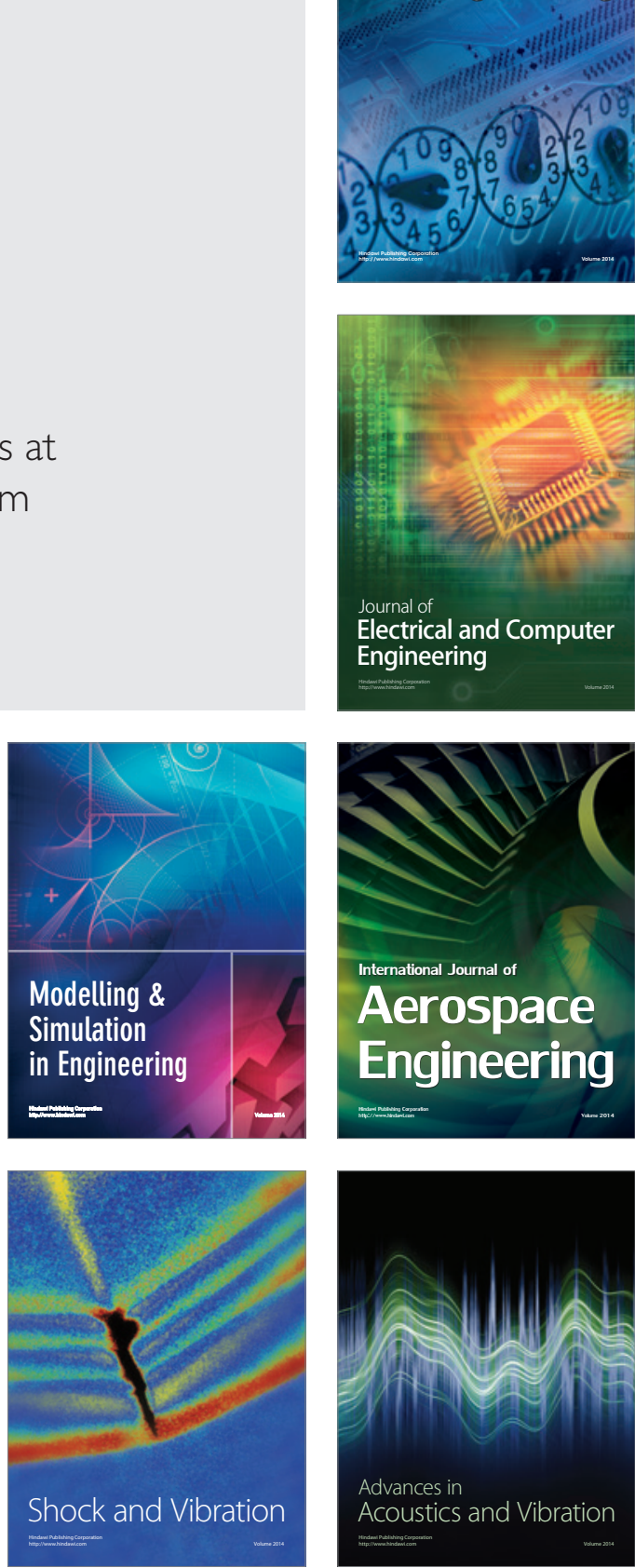